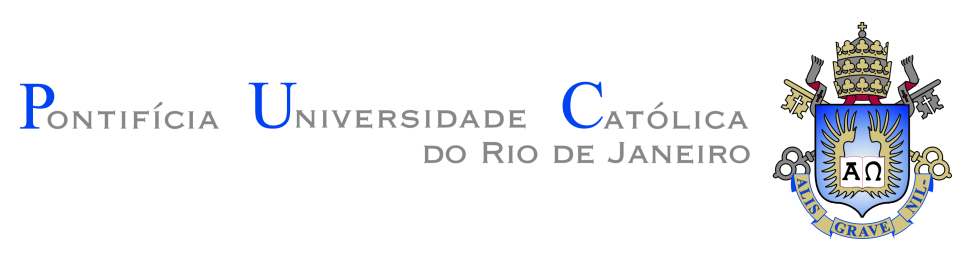

André Pimentel de Oliveira

\title{
Aplicação da derivada topológica na otimização \\ estrutural
}

\author{
Dissertação de Mestrado
}

Dissertação apresentada como requisito parcial para obtenção do grau de Mestre pelo Programa de Pós-graduação em Engenharia Mecânica do Departamento de Engenharia Mecânica do Centro Técnico Científicoda PUC-Rio.

Orientador : Prof. Ivan Fabio Mota de Menezes Co-orientador: Prof. Rafael Menezes de Oliveira 


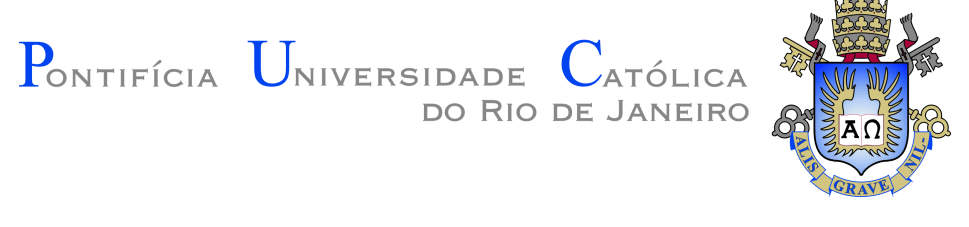

André Pimentel de Oliveira

\section{Aplicação da derivada topológica na otimização estrutural}

Dissertação apresentada como requisito parcial para obtenção do grau de Mestre pelo Programa de Pós-graduação em Engenharia Mecânica do Departamento de Engenharia Mecânica do Centro Técnico Científicoda PUC-Rio. Aprovada pela Comissão Examinadora abaixo assinada.

Prof. Ivan Fabio Mota de Menezes

Orientador

Departamento de Engenharia Mecânica - PUC-Rio

Prof. Rafael Menezes de Oliveira

Co-orientador

Departamento de Engenharia Mecânica - PUC-Rio

Prof. Anderson Pereira

Departamento de Engenharia Mecânica - PUC-Rio

Dr. Djalma Manoel Soares Filho

Centro de Desenvolvimento e Pesquisas (CENPES) - Petrobras

Prof. Márcio da Silveira Carvalho

Coordenador Setorial do Centro

Técnico Científico - PUC-Rio

Rio de Janeiro, 25 de Outubro de 2018 
Todos os direitos reservados. É proibida a reprodução total ou parcial do trabalho sem autorização da universidade, do autor e do orientador.

\section{André Pimentel de Oliveira}

O autor se formou em Engenharia Mecânica na Pontifícia Universidade Católica do Rio de Janeiro (PUC-Rio) em Julho de 2016.

Ficha Catalográfica

Oliveira, André Pimentel de

Aplicação da derivada topológica na otimização estrutural / André Pimentel de Oliveira; orientador: Ivan Fabio Mota de Menezes; co-orientador: Rafael Menezes de Oliveira. - 2018.

58 f: il. color. ; $30 \mathrm{~cm}$

Dissertação (mestrado) - Pontifícia Universidade Católica do Rio de Janeiro, Departamento de Engenharia Mecânica, 2018.

Inclui bibliografia

1. Engenharia Mecânica - Teses. 2. Derivada topológica. 3. Elasticidade linear. 4. Elementos poligonais. 5. Otimização topológica. 6. SIMP. I. Menezes, Ivan Fabio Mota de. II. Oliveira, Rafael Menezes de. III. Pontifícia Universidade Católica do Rio de Janeiro. Departamento de Engenharia Mecânica. IV. Título. 


\section{Agradecimentos}

Agradeço antes de tudo, a Deus, por todas as oportunidades e pessoas que Ele tem colocado no meu caminho, que permitiram a realização deste curso de Mestrado. Nisso se inclui todo o apoio de minha família, em especial de minha mãe Marcia e minha irmã Natália, que têm estado ao meu lado dando muito suporte, com muita paciência, carinho e amor necessários nesta longa trajetória.

Ao meu pai, Ricardo, pelo orgulho que sempre teve de mim.

Ao grande e especial amigo Alcides, que tem estado comigo nos momentos mais difíceis.

Ao meu coorientador, Rafael Menezes, sempre tão presente em cada fase deste trabalho e que, mais que tudo, se tornou um verdadeiro amigo nessa trajetória.

Ao meu orientador, Ivan Menezes, por me propor este desafio e por sempre ter confiado na minha capacidade em realizá-lo.

A uma pessoa muito especial, sempre presente, Dra. Míria Amorim.

E a todos e tantos amigos que sempre me apoiaram com a sua presença ou nos momentos de descontração na internet, entre eles, Brenner, Thomaz, Alexandre, Leo, Felipes, Thiago, Edson, Jefferson, João, Gabriel, Henrique. 


\section{Resumo}

Oliveira, André Pimentel de; Menezes, Ivan Fábio Mota de; Oliveira, Rafael Menezes de. Aplicação da derivada topológica na otimização estrutural. Rio de Janeiro, 2018. 58p. Dissertação de Mestrado - Departamento de Engenharia Mecânica, Pontifícia Universidade Católica do Rio de Janeiro.

A otimização topológica tem por objetivo buscar uma distribuição ótima de uma quantidade limitada de material em um dado domínio, de tal maneira a minimizar uma medida de desempenho, como, por exemplo, a flexibilidade da estrutura. Tradicionalmente, são utilizados algoritmos clássicos, baseados em gradiente, para se encontrar a solução deste problema de otimização. Este trabalho propõe a aplicação de uma técnica alternativa, baseada no conceito de derivada topológica, para a solução do problema de otimização topológica em domínios bidimensionais arbitrários, utilizando malhas de elementos finitos poligonais. Inicialmente, são apresentados os conceitos básicos da expansão assintótica topológica na solução de problemas de elasticidade linear em um domínio com pequenas perturbações. Usamos esse conceito para definir a derivada topológica a partir da solução desse problema e de um equivalente em um domínio sem perturbações. Em seguida, discutimos a obtenção da derivada topológica em problemas unidimensionais simples para depois estender este conceito para problemas de elasticidade linear bidimensional. Apresentamos uma implementação computacional da derivada topológica, em MATLAB, e aplicamos o código desenvolvido na solução de problemas de otimização topológica, conhecidos na literatura. Finalmente, apresentamos as conclusões sobre a qualidade dos resultados obtidos e a eficiência computacional da implementação proposta e sugerimos alguns tópicos para futuros desenvolvimentos.

\section{Palavras-chave}

Derivada topológica; Elasticidade Linear; Elementos Poligonais; Otimização topológica; SIMP. 


\section{Abstract}

Oliveira, André Pimentel de; Menezes, Ivan Fábio Mota de (Advisor); Oliveira, Rafael Menezes de (Co-Advisor). Application of topological derivative in structural optimization. Rio de Janeiro, 2018. 58p. Dissertação de Mestrado - Departamento de Engenharia Mecânica, Pontifícia Universidade Católica do Rio de Janeiro.

The purpose of topology optimization is to find the optimum material distribution of a limited amount of material in a given domain, in such a way that it minimizes a performance measure, such as the structure's compliance. Traditionally, classical algorithms based on gradients are used to obtain the solution of optimization problems. This work proposes the application of an alternative technique, based on the topological derivative concept, for the solution of topology optimization problems in arbitrary two-dimensional domains, using polygonal finite element meshes. Initially, the basic concepts of topological asymptotic expansion of linear elasticity problems in a domain with small perturbations are presented. We use this concept to define the topological derivative from the solution of this problem and an equivalent one on a domain without perturbations. Then, we discuss how to calculate the topological derivative for one-dimensional problems before extending this concept to two-dimensional linear stability problems. We present a computational implementation of the topological derivative in MATLAB, and apply the developed code to solve topology optimization problems known in the literature. Finally, we present some conclusions about the quality of the results obtained and the computational efficiency of the proposed implementation and suggest some topics for future developments.

\section{Keywords}

Topology derivative; Polygonal elements; Linear elasticity; Topological optimization; SIMP. 


\section{Sumário}

1 Introdução 11

1.1 Otimização Topológica 12

1.2 Objetivo 13

2 A Derivada Topológica $r$

2.1 Introdução 15

2.2 Comparação com a Série de Taylor 19

$\begin{array}{ll}2.3 \text { Exemplos } & 22\end{array}$

3 Cálculo da derivada topológica para o problema de elasticidade $\quad 29$

3.1 Formulação 29

3.2 Análise Assintótica 33

3.3 Cálculo da tensão 35

3.4 Determinação do funcional da energia interna do sistema 37

4 Implementação da Derivada Topológica 41

4.1 Elementos Quadrilaterais x Elementos Poligonais 41

4.2 Implementação $\quad 45$

5 Resultados $\quad 50$

5.1 Metodologia 50

5.2 MBB Domain $\quad 51$

5.3 Hook Domain 52

5.4 Serpentine Domain $\quad 53$

6 Conclusões $\quad 55$

$\begin{array}{ll}\text { Referências bibliográficas } & 56\end{array}$ 


\section{Lista de figuras}

Figura 1.1 Principais abordagens da otimização estrutural: (a) Otimização Paramétrica; (b) Otimização de Forma; (c) Otimização Topológica Fonte: [3]

Figura 1.2 a) Um escritório no Japão com paredes otimizadas; b) Um suporte de estrutura otimizado; c) Uma marquise em Doha otimizada; Fonte: [10]

Figura 2.1 Definição de domínio com perturbação.

Figura 2.2 a) Domínio com $\gamma=0$; b) Domínio com $\gamma \neq 0$

Figura 2.3 (a) Metade de uma viga bi-apoiada com uma força no centro; (b) Primeira iteração

Figura 2.4 Série de Taylor de primeira ordem para a função $f(x)=$ $\ln (x)$, em torno do ponto $x=2$

Figura 2.5 Intervalo da perturbação

Figura 2.6 Aumento do erro com o aumento da perturbação

Figura 2.7 Erro percentual com o aumento da perturbação

Figura 3.1 Problema de elasticidade: Estado plano de tensões segundo Novotny [8]

Figura 4.1 Exemplo de baixa ordem de elementos: (a) quadrilaterias; e (b) triangulares

Figura 4.2 Problema de checkerboard; Fonte: [28]

Figura 4.3 Comparação entre (a) elementos de quatro nós e (b) elementos de oito nós; Fonte: [30]

Figura 4.4 Malha gerada com 400 elementos usando o código Polytop [19]

Figura 4.5 Problema One-Node Connection

Figura 4.6 (a) área dos triângulos; (b) sistema de coordenadas. Fonte: [19]

Figura 5.1 (a) Malha utilizada para a realização da otimização topológica; (b) Topologia final obtida após $p=4$.

Figura 5.2 (a) Malha utilizada para a realização da otimização topológica; (b) Topologia final obtida após $p=4$.

Figura 5.3 Domínio serpentine [31]

Figura 5.4 (a) Malha utilizada para a realização da otimização topológica; (b) Topologia final obtida após $p=4$. 


\section{Lista de tabelas}

Tabela 2.1 Erros da série de Taylor 


\section{Lista de Abreviaturas}

c - Função compliance

$\mathrm{f}$ - Vetor de forças atuantes no sistema

$V_{i}$ - Volume de cada elemento da malha

$V_{s}$ - Volume desejado

$\boldsymbol{K}$ - Matriz de rigidez global

$\boldsymbol{u}$ - Vetor de deformações do domínio contínuo

$\boldsymbol{u}_{\boldsymbol{\epsilon}}-$ Vetor de deformações do domínio perturbado

$\chi_{\epsilon}$ - Solução do sistema avaliada em um funcional com perturbação

$\chi$ - Solução do sistema avaliada em um funcional sem perturbação

$\epsilon$ - Função do tamanho da perturbação

$\Gamma$ - Função da derivada topológica

$\gamma$ - Densidade do material

$a$ - Ponto da função

$\Omega$ - Domónio contínuo

$\Omega_{\epsilon}-$ Domínio perturbado

$w_{\epsilon}$ - Domínio que contém a perturbação

$\Gamma_{D}-$ Região com condição de Dirichlet

$\Gamma_{N}-$ Região com dondição de Neumann

$\partial \Omega$ - Fronteira de um domínio

$\boldsymbol{\sigma}$ - Tensões atuantes em um domínio contínuo

$\boldsymbol{\sigma}_{\boldsymbol{\epsilon}}$ - Tensões atuantes em um domínio perturbado

$\mathbb{C}$ - Tensor constitutivo

$\boldsymbol{I}$ - Tensor identidade de segunda ordem

0 - Tensor identidade de quarta ordem

$\mu$ - Coeficiente de Lamé

$\lambda$ - Coeficiente de Lamé

$\nu$ - Coeficiente de Poisson

E - Módulo de Young

$\boldsymbol{n}$ - Vetor normal ao plano

$\mathbb{T}$ - Tensor uniforme de quarta ordem

$\boldsymbol{D}$ - Matriz de elasticidade

$\boldsymbol{N}$ - Função de forma 


\section{1 \\ Introdução}

Um dos maiores objetivos da engenharia na área estrutural é reduzir ao máximo a quantidade de material, tal que a estrutura consiga suportar cargas pré definidas. Para que esses avanços sejam possíveis, é fundamental a criação de um modelo ótimo que descreva o comportamento mecânico do sistema. Uma área onde esses avanços vêm sendo observados é a área da otimização estrutural. Para o melhor entendimento do conceito de otimização estrutural, segundo a literatura, podemos dividi-la em três grandes áreas:

- Otimização Paramétrica;

- Otimização de Forma;

- Otimização Topológica.

Na otimização paramétrica a topologia da estrutura final já é conhecida, e seu objetivo é tentar otimizar as dimensões de seus componentes, onde as incógnitas podem ser, por exemplo, os diâmetros das barras que compõem a estrutura ou a espessura de uma chapa metálica. Vários métodos podem ser utilizados para resolver esse tipo de problema, como os Algoritmos Genéticos $[1,2]$, nos quais são criados indivíduos que representam uma solução potencial do problema e, em seguida, alguns destes são escolhidos para gerar melhores descendentes (valores) que irão cada vez mais se aproximar do resultado desejado.

$\mathrm{Na}$ otimização de forma, tenta-se modificar a geometria dos contornos externos e internos (exemplo: furos pré existentes), de maneira a maximizar alguma medida de desempenho da estrutura, como por exemplo, reduzir minimizar a energia interna do sistema.

E por fim, a otimização topológica, que é o foco deste trabalho. Esta abordagem baseia-se em: a partir de um dado domínio, procurar-se a melhor distribuição de material neste espaço tal que otimize alguma medida de desempenho, como a minimização da flexibilidade da estrutura, e que leve à redução desejada no volume. A Figura 1.1 ilustra cada um dos três tipos de otimização. 

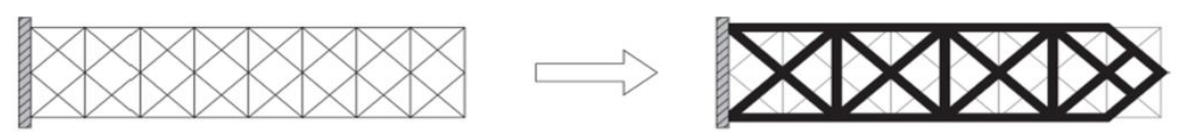

(a)
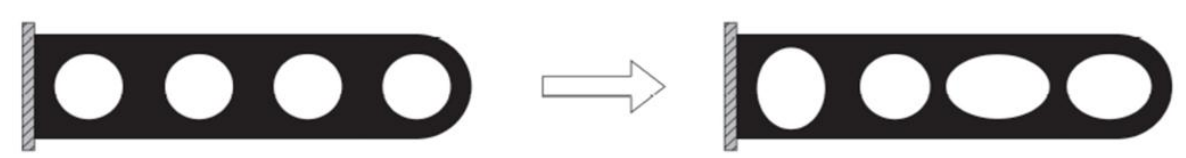

(b)
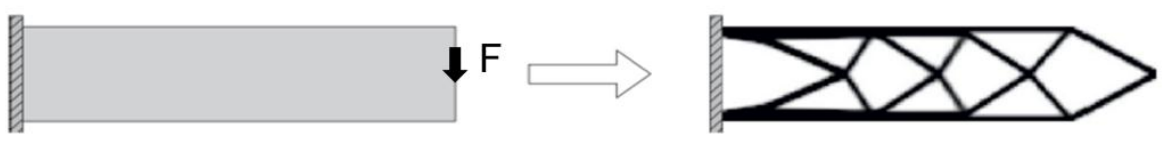

(c)

Figura 1.1: Principais abordagens da otimização estrutural: (a) Otimização Paramétrica; (b) Otimização de Forma; (c) Otimização Topológica Fonte: [3]

O problema típico de otimização topológica pode ser descrito como a minimização da flexibilidade ${ }^{1}$ da estrutura, ou seja:

$$
\begin{cases}\min _{\boldsymbol{x}} & \boldsymbol{c}(\boldsymbol{x})=\boldsymbol{q}^{T} \boldsymbol{u} \\ \text { Sujeito a }: & \\ & \sum_{i}^{N_{e l}} V_{i} x_{i}-V_{s} \leq 0 \\ \text { Com: } & \boldsymbol{K u}=\boldsymbol{q} \\ x_{i} \in[0,1], & i=1, \ldots, N_{e l}\end{cases}
$$

onde $\boldsymbol{K} \boldsymbol{u}=\boldsymbol{q}$ é a equação de equilíbrio, $\boldsymbol{K}$ a matriz de rigidez, $\boldsymbol{u}$ é o vetor dos deslocamentos nodais e $\boldsymbol{q}$ o vetor de forças aplicadas ao sistema, tentando minimizar $\boldsymbol{c}(\boldsymbol{x})$ tal que o volume final dado pela soma dos volumes de cada elemento da malha $V_{i}$, totalizando um número total de elementos $N_{e l}$, não seja maior que o volume desejado $V_{s}$.

\section{1}

\section{Otimização Topológica}

$\mathrm{Na}$ otimização topológica, existem diferentes algoritmos de otimização que podem ser utilizados para a minimização da compliance. $\mathrm{O}$ método $\mathrm{OC}$ (Optimality Criteria) é um dos mais utilizados para solução de problemas de

${ }^{1}$ Em inglês, a flexibilidade é conhecida como compliance. 
otimização topológica [4]. Pode-se também usar o Sequential Linear Programming [5] (SLP), que para obter a solução ótima, resolve uma sequência de aproximações de primeira ordem do problema de otimização.

Há ainda um outro método chamado level set [6,7,8,9], onde representamse as regiões do domínio que devem ser preenchidas com material e quais não através de uma equação diferencial. Esta região é atualizada de acordo com a equação diferencial que precisa ser resolvida com o objetivo de minimizar uma determinada função objetivo. Exemplos de aplicação da otimização topológica são ilustrados na Figura 1.2.
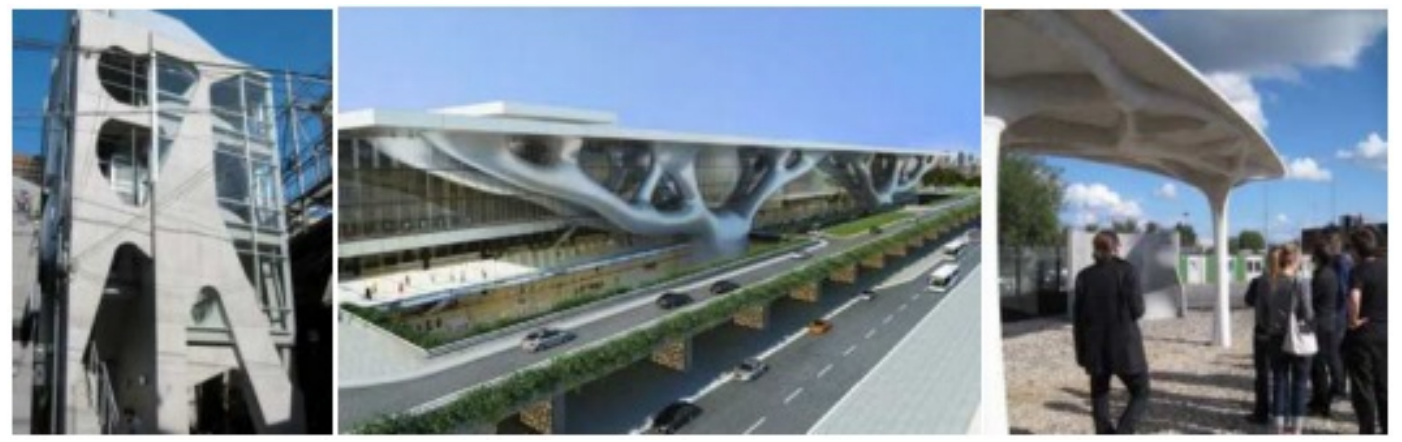

Figura 1.2: a) Um escritório no Japão com paredes otimizadas; b) Um suporte de estrutura otimizado; c) Uma marquise em Doha otimizada; Fonte: [10]

Neste trabalho, será apresentada um solução alternativa, já existente, para a otimização topológica baseada no conceito de derivada topológica. Trata-se de um método onde a compliance é definida pela grandeza derivada topológica.

\section{2}

\section{Objetivo}

A teoria sobre a derivada topológica foi desenvolvida por Sokolowski e Zochowski [11] e foi utilizada por vários autores como, Novotny [12], Suresh [13] e Otomori [14], onde é comumente implementada com elementos quadrilaterais. Matematicamente, esta teoria necessita de explicações e detalhamentos para ser melhor compreendida. Dessa forma, os objetivos específicos deste trabalho são:

(a) Explicar de forma clara o conceito de derivada topológica;

(b) Expandir este conceito para a otimização topológica mostrando os desenvolvimentos matemáticos necessários;

(c) Utilizar elementos finitos não convencionais para melhorar os resultados obtidos; 
(d) Fazer uma análise detalhada deste método para verificar a sua eficiência e adequabilidade em relação às técnicas tradicionais utilizadas na otimização topológica. 


\section{2}

\section{A Derivada Topológica}

Neste capítulo, serão apresentados a definição do conceito de derivada topológica e os fatores que irão resultar no seu desenvolvimento matemático. Para que o leitor compreenda melhor este conceito, será utilizado o conceito de aproximação de funções por séries de Taylor para fazer uma analogia com a derivada topológica. Em seguida, serão apresentados exemplos para demonstrar a simplicidade do conceito da Derivada Topológica.

\section{1}

\section{Introdução}

A derivada topológica define a influência de uma perturbação em um determinado ponto de um domínio para um determinado funcional. Por exemplo, na otimização topológica pequenos furos são criados com objetivo de remover material e identificar a forma final da estrutura, criando uma topologia ótima. Existem dois conceitos fundamentais para se entender o método empregado neste trabalho:

- Derivada Topológica

- Expansão Assintótica Topológica.

A derivada topológica é uma grandeza que expressa a influência de uma pequena perturbação em um domínio, enquanto que a expansão assintótica topológica é a equação que irá dizer quem é a derivada topológica para um determinado problema que possui um funcional. A expansão assintótica topológica é definida da seguinte forma:

$$
\psi\left(\chi_{\epsilon}(\hat{x})\right)=\psi(\chi(\hat{x}))+f(\epsilon) \Gamma(\hat{x})+o^{2}(f(\epsilon)) \quad,
$$

onde o termo referente a derivada topológica é dado por $\Gamma(\widehat{x})$. Para calcular o seu valor são necessárias três informações: a função da perturbação $f(\epsilon)$, que varia dependendo do subespaço do $\mathbb{R}^{n}$ em que a solução do problema está contida; o valor que o funcional a ser resolvido possui no domínio contínuo sem a presença de perturbações $\psi(\chi(\hat{x}))$, com $\chi(\hat{x})$, sendo a solução do funcional em domínio contínuo; e uma forma que permita encontrar o valor do funcional 
do domínio com as perturbações presentes $\psi\left(\chi_{\epsilon}(\hat{x})\right)$, sendo $\chi_{\epsilon}(\hat{x})$ a solução do funcional em domínio perturbado. Para se obter a expansão assintótica topológica é necessário avaliar a influência da perturbação em seu centro, pois como ela é muito pequena, com seu tamanho $\epsilon \rightarrow 0$, o erro será desprezível e irá gerar resultados próximos e satisfatórios. A equação do furo $f(\epsilon)$, é comumente escrita da seguinte forma:

$$
f(\epsilon)= \begin{cases}\epsilon, & \text { para } \mathbb{R}^{1} \\ \pi \epsilon^{2}, & \text { para } \mathbb{R}^{2} \\ \frac{4}{3} \pi \epsilon^{3}, & \text { para } \mathbb{R}^{3}\end{cases}
$$

ou seja, um segmento de reta de tamanho $\epsilon$, ou um círculo de raio $\epsilon$ ou uma esfera de raio $\epsilon$, dependendo em que espaço o problema se encontra, sempre considerando pequenas perturbações.

Para escrever o problema na forma da expansão assintótica topológica, é necessário resolver o sistema de equações que definem, por exemplo, o valor de $\boldsymbol{x}$. Em seguida, avaliam-se os funcionais $\psi\left(\chi_{\epsilon}(\hat{x})\right)$ e $\psi(\chi(\hat{x}))$ a partir deste resultado. Pela Eq. (2.1.1), observa-se que a diferença entre estes dois funcionais é a função do furo descrita pela Eq. (2.1.2) multiplicada pela derivada topológica, ou seja, é necessário apenas descobrir a solução do domínio para se calcular a derivada topológica.

Um domínio $\Omega$ a ser trabalhado pode ser definido como ilustrado na Figura 2.1.

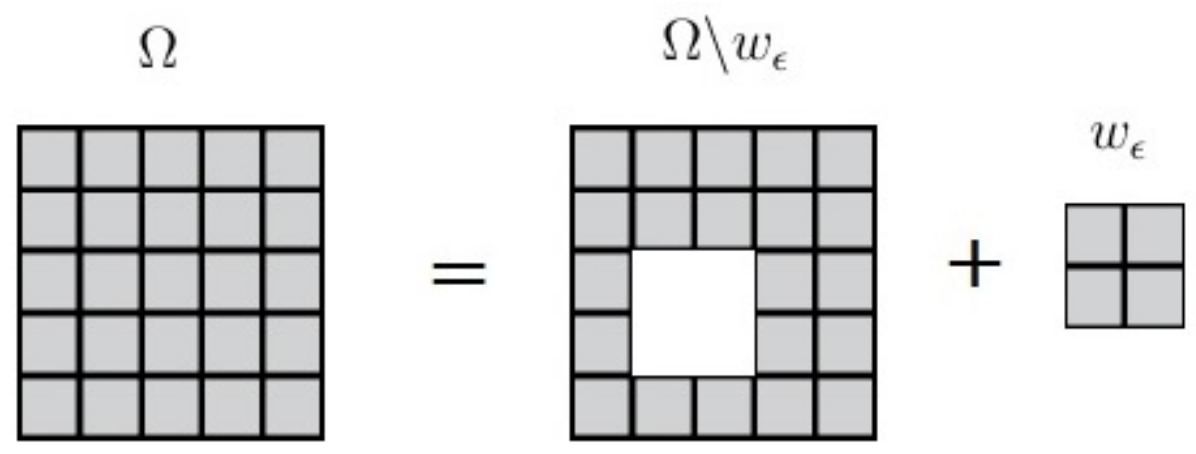

Figura 2.1: Definição de domínio com perturbação.

O funcional associado ao domínio não perturbado deve ser igual ao funcional do domínio perturbado acrescido do furo, ou seja: $\Omega=\Omega \backslash w_{\epsilon}+w_{\epsilon}$. Observando a forma da expansão assintótica topológica, pode-se concluir que uma vez calculado o valor do funcional do domínio perturbado o valor da derivada topológica pode ser facilmente obtido. 
Se a perturbação não for um furo, mas sim a substituição de um material já existente por outro de densidade diferente $\gamma$ (região de cor cinza mais escuro da Figura 2.2b), é necessário que a densidade deste novo material seja considerada, pois, caso contrário, volta-se ao primeiro caso em que a perturbação é apenas um furo no domínio.
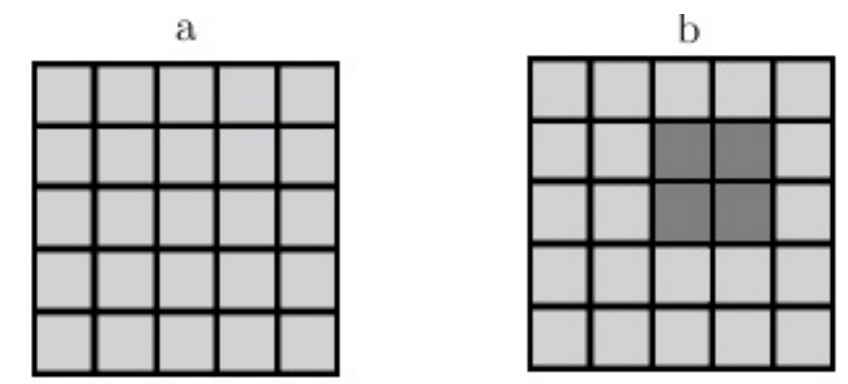

Figura 2.2: a) Domínio com $\gamma=0$; b) Domínio com $\gamma \neq 0$

$\mathrm{Na}$ otimização topológica, existem tons de cinza que representam a variação de densidade do material na região, conforme ilustrado na Figura 2.3b. Os extremos, preto e branco são, respectivamente, áreas com material de densidade $\gamma=1$ e $\gamma=0$ (sem material), estes tons de cinza vão sendo eliminados à medida que o processo iterativo vai sendo realizado. Pode-se também empregar métodos baseados em curvas de nível (level-set), de forma que estas densidades intermediárias não aparecem. Considere a otimização de uma viga bi-apoiada com uma carga concentrada no centro, conforme ilustrado na Figura 2.3a, onde apenas metade da viga está sendo considerada, por questões de simetria. 


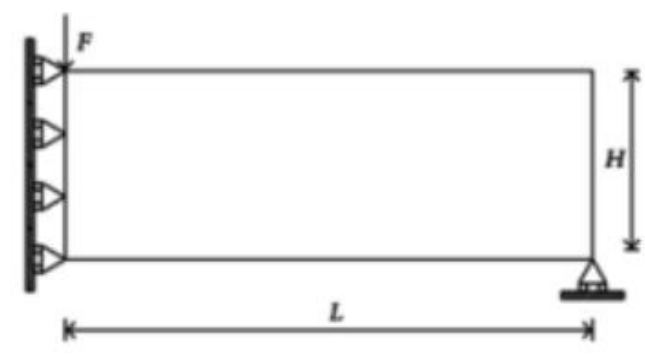

(a)

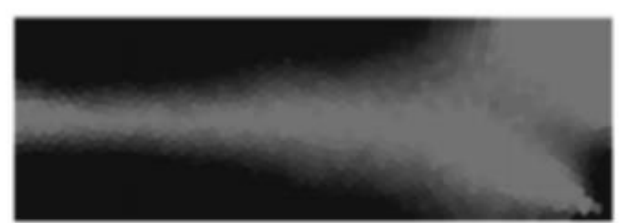

(b)

Figura 2.3: (a) Metade de uma viga bi-apoiada com uma força no centro; (b) Primeira iteração

Calculando-se a solução do problema de otimização topológica, a expansão assintótica topológica pode ser obtida por meio da diferença de funcionais. Desta forma, é possível obter a função que define a derivada topológica, a qual apresentará valores diferentes para cada elemento, porém, como existem densidades intermediárias de $0 \leq \gamma \leq 1$, o problema se torna parecido com o da Figura 2.3b, i.e., a derivada topológica, que será calculada mais adiante, irá fornecer o vetor de densidades da malha. 


\section{2}

\section{Comparação com a Série de Taylor}

A série de Taylor é uma aproximação que se assemelha em forma à derivada topológica. Considerando $f(x)$ uma função $f: \mathbb{R} \rightarrow \mathbb{R}$, contínua e diferenciável, uma aproximação $S(x)$ de $f(x)$ em torno de um ponto $x=a$ pode ser obtido a partir da Série de Taylor, cujos primeiros termos são dados por:

$$
S(x)=f(a) \frac{(x-a)^{0}}{0 !}+f^{\prime}(a) \frac{(x-a)^{1}}{1 !}+o^{2}(a)
$$

Quando se trabalha com o conceito da série de Taylor, a aproximação dita de primeira ordem em torno de um determinado ponto a, é calculada conhecendo-se dois valores: $f(a)$, o valor da função no ponto e $f^{\prime}(a)$, a derivada da função no ponto. Observando a expansão assintótica topológica dada pela Eq. (2.1.1), percebe-se que para se calcular a derivada topológica também é necessário conhecer dois valores: $\psi\left(\chi_{\epsilon}(\hat{x})\right)$, o valor do funcional da solução de um problema definido em um domínio perturbado e $\psi(\chi(\hat{x}))$, o valor do funcional da solução de um problema definido em um domínio contínuo.

Quanto mais longe estivermos do ponto calculado na série de Taylor, maior será o erro na aproximação da função. Analogamente, quanto maior for a dimensão do furo $\epsilon$ na expansão assintótica topológica, maior será o erro na aproximação realizada para a solução do problema definido em um domínio perturbado.

Considere um exemplo simples para mostrar essa analogia, e que servirá de base para o Exemplo 1 do próximo tópico. Fazendo a expansão em série de Taylor de primeira ordem da função $f(x)=\ln (x)$, em torno do ponto $x=2$, tem-se o seguinte gráfico: 


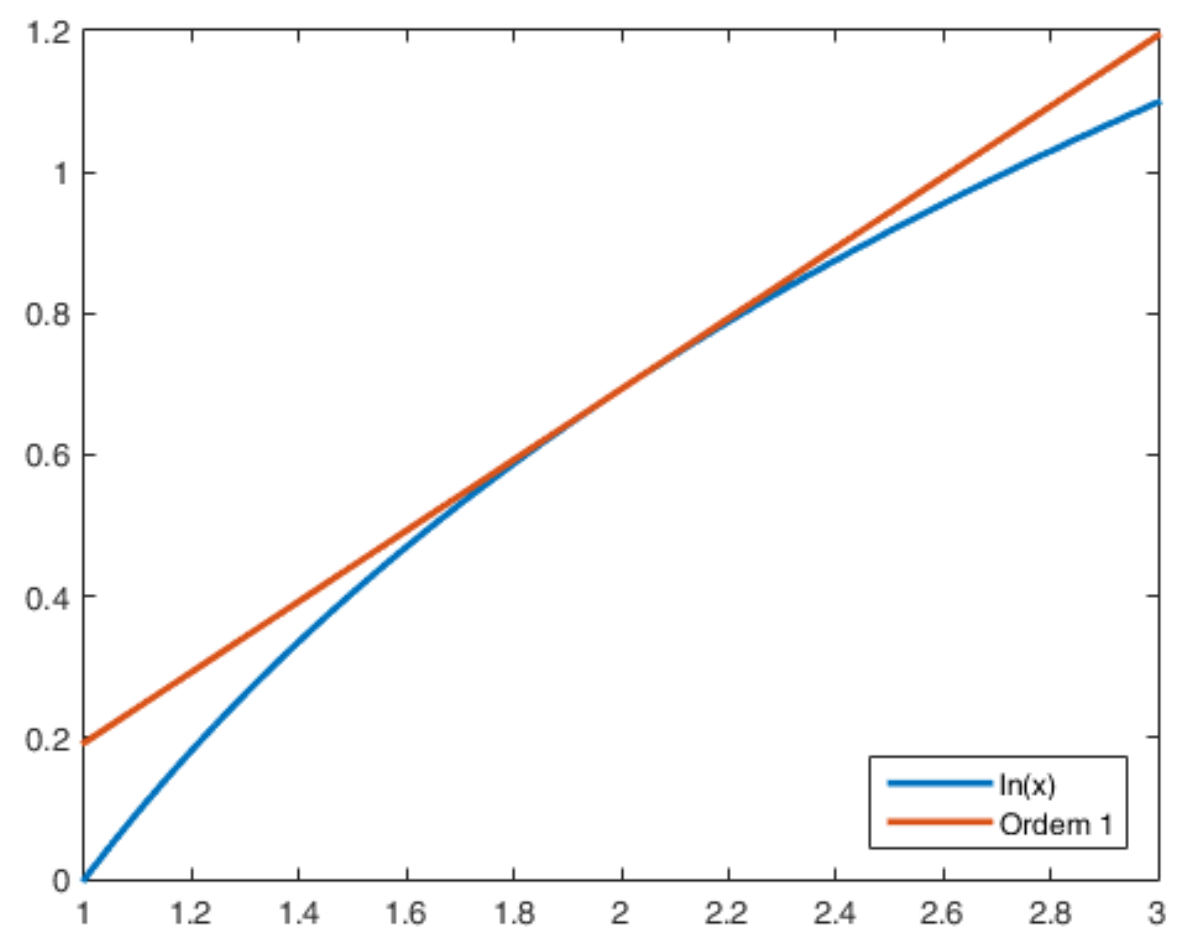

Figura 2.4: Série de Taylor de primeira ordem para a função $f(x)=\ln (x)$, em torno do ponto $x=2$

A Tabela 1 apresenta valores de pontos próximos e afastados do ponto de referência e os respectivos erros, enquanto que a Figura 2.5 representa a função a ser avaliada e sua aproximação de primeira ordem. 
Tabela 2.1: Erros da série de Taylor

\begin{tabular}{||cccc||}
\hline Ponto & Exato & Aproximado & Erro \% \\
\hline \hline $\mathbf{1 , 1}$ & 0,0953 & 0,2431 & 255,11 \\
\hline $\mathbf{1 , 5}$ & 0,4055 & 0,4431 & 9.27 \\
\hline $\mathbf{1 , 9}$ & 0,6419 & 0,6432 & 0,85 \\
\hline $\mathbf{2 , 1}$ & 0,7419 & 0,7431 & 0,85 \\
\hline $\mathbf{2 , 5}$ & 0,9163 & 0,9431 & 5,73 \\
\hline $\mathbf{3 , 0}$ & 1,0986 & 1,1931 & 10,37 \\
\hline
\end{tabular}

Conforme esperado, quanto maior a distância ao ponto de referência, maior será o erro na aproximação de $f(x)$. Na próxima seção, serão mostrados exemplos de como se chega a expansão assintótica topológica e como esta expansão se assemelha a uma expansão em série de Taylor. 


\section{3}

\section{Exemplos}

\section{- Exemplo 1}

O primeiro exemplo consiste no cálculo da derivada topológica da integral de uma função em um domínio com uma perturbação.

$$
\psi\left(\chi_{\epsilon}\right)=\int_{\Omega_{\epsilon}} g(x)
$$

O que se procura é escrever o problema na forma da expansão assintótica topológica. O domínio perturbado, segundo a Figura 2.1, é $\Omega_{\epsilon}=\Omega-w_{\epsilon}$, assim, soma-se e subtrai-se o furo para resultar no domínio contínuo e não alterar a equação original de forma que:

$$
\psi\left(\chi_{\epsilon}\right)=\int_{\Omega_{\epsilon}} g(x)+\int_{w_{\epsilon}} g(x)-\int_{w_{\epsilon}} g(x) .
$$

Juntando os termos $\Omega_{\epsilon}$ e $w_{\epsilon}$ :

$$
\psi\left(\chi_{\epsilon}\right)=\int_{\Omega} g(x)-\int_{w_{\epsilon}} g(x)
$$

O exemplo apresentado encontra-se no espaço $\mathbb{R}^{1}$, em que a perturbação é um intervalo de tamanho $\epsilon$. No lado esquerdo da Eq. (2.3.3), observa-se o funcional do domínio perturbado e, no lado direito, o funcional do domínio contínuo menos um termo que corresponde a uma integral da perturbação. Assim, pode-se aproximar o valor desta integral pelo tamanho da perturbação vezes o valor da função avaliada no centro desta perturbação, pois como a perturbação é pequena, ou seja, $\epsilon \rightarrow 0$, é possível utilizar o teorema do valor médio sem que haja grandes erros. Logo a Eq. (2.3.3) pode ser reescrita como:

$$
\int_{\Omega_{\epsilon}} g(x)=\int_{\Omega} g(x)-\left|w_{\epsilon}\right| g(\hat{x})
$$

Comparando-se com a expansão assintótica dada pela Eq. (2.1.1), podese concluir que a derivada topológica é $\Gamma(\hat{x})=g(\hat{x})$.

Fazendo-se: $f(x)=x^{2}$, deseja-se agora saber qual a área correspondente nos intervalos de $x=0$ a $x=4,8$ e $x=5,2$ e $x=10,0$, ou seja, há uma perturbação entre $x=4,8$ e $x=5,2$ de tamanho 0,4 . Este intervalo é ilustrado na Figura 2.5. 


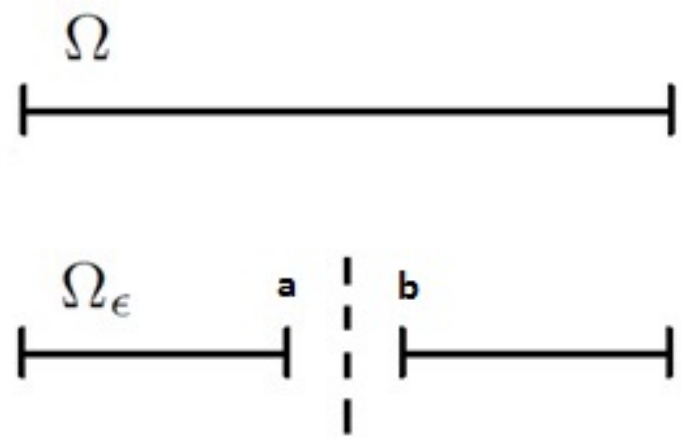

Figura 2.5: Intervalo da perturbação

A solução exata para este problema é obtida da seguinte forma:

$$
\psi_{\epsilon}=\int_{0}^{4,8} x^{2} d x+\int_{5,2}^{10,0} x^{2} d x=323,3280
$$

Agora, aplicando-se a derivada topológica, tem-se por meio da Eq. (2.3.4), onde $w_{\epsilon}$ é o tamanho da pertubação, $0,4, g(\hat{x})$, o valor da função no centro do furo $f(5,0)$ e a integral no domínio contínuo de $x=0$ a $x=10$, 0 . Substituindose estes valores:

$$
\psi_{\epsilon}=\int_{0}^{10,0} x^{2} d x-0,4 \times 5,0^{2}=323,3333 .
$$

Neste caso, pode-se observar que, mesmo com uma perturbação grande, o erro é relativamente baixo. Os gráficos apresentados nas Figuras 2.6 e 2.7 mostram a variação do erro para a mesma função com um domínio compreendido de $x=0$ a $x=10$, a perturbação centrada em $x=5,0$, e aumentado-se esta perturbação igualmente para os dois lados de modo que seu ponto central não se altere. 


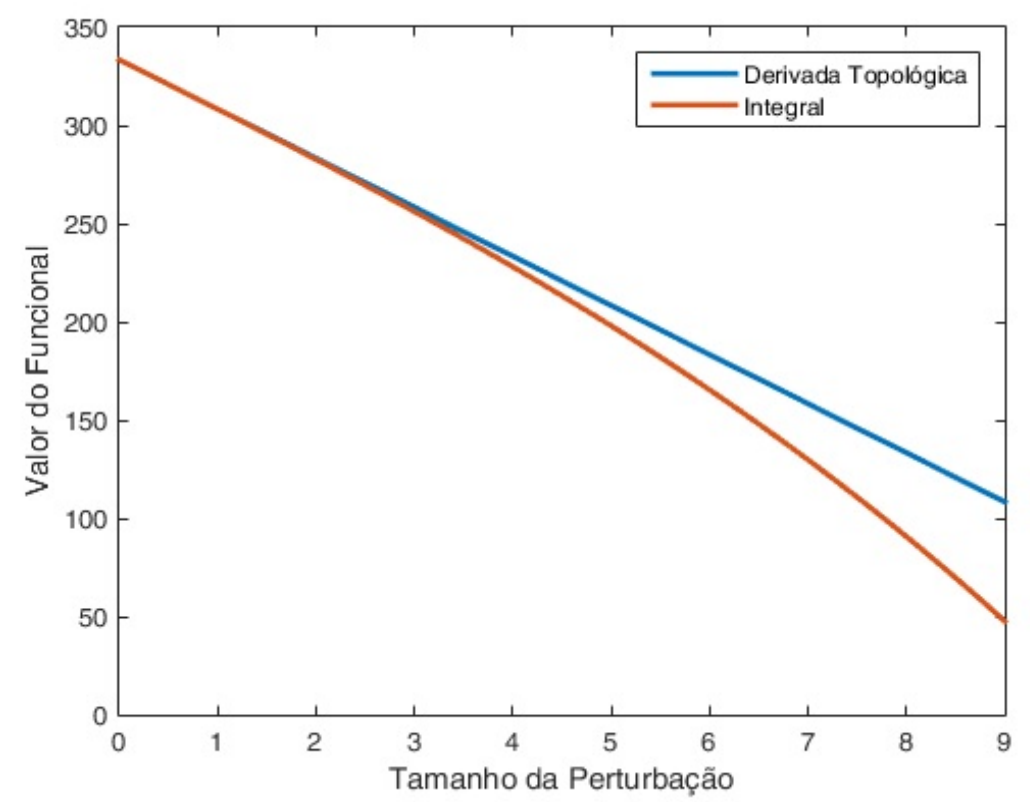

Figura 2.6: Aumento do erro com o aumento da perturbação

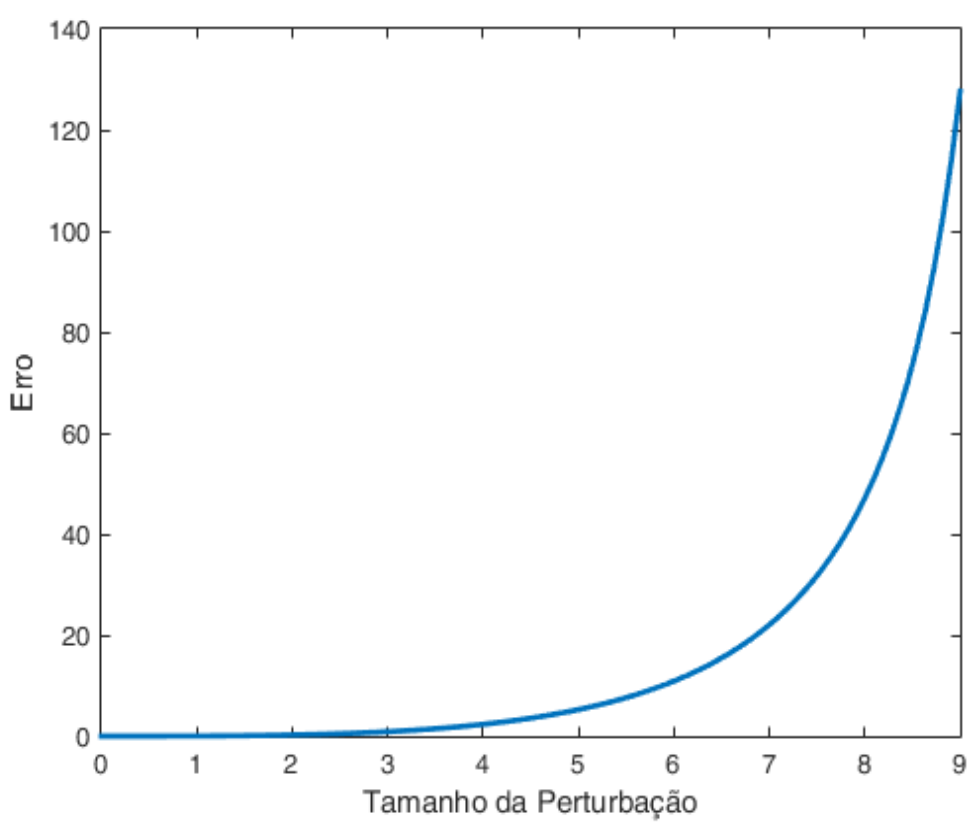

Figura 2.7: Erro percentual com o aumento da perturbação

De acordo com os gráficos das Figuras 2.7 e 2.8, pode-se concluir que, a partir de um determinado valor para o tamanho $\epsilon$, o erro começa a aumentar e, a medida em que o furo diminui $(\epsilon \rightarrow 0)$, o resultado passa a ser muito próximo da solução exata. Assim como na série de Taylor, distâncias grandes do ponto de referência, ou, furos grandes, implicam em erros maiores. 
- Uma questão interessante a ser abordada é qual seria então a vantagem em se aplicar um método aproximado no lugar do exato?

No método exato, cada vez que se altera a posição do furo ou o tamanho do furo é necessário recalcular ambas as integrais para se obter o valor do funcional do novo domínio. Enquanto que no caso da derivada topólógica, uma vez calculado o valor do funcional do domínio contínuo, o único passo necessário é mudar o valor do tamanho da perturbação e do centro do mesmo, ou seja, trata-se um processo muito mais rápido e simples.

Neste primeiro exemplo, o cálculo da derivada topológica foi relativamente fácil, o que não é algo recorrente. No próximo exemplo a ser apresentado, que também irá ajudar na seção sobre otimização topológica, será mostrado que nem sempre este processo de se chegar em um modelo no mesmo formato da Eq. (2.1.1) é simples, porém, uma vez calculado o seu resultado, a metodologia é mais fácil de ser aplicada.

\section{- Exemplo 2}

Este exemplo apresenta, um caso em que a perturbação aparece na função devido a um contraste de densidade $\gamma$ :

$$
\psi\left(\chi_{\epsilon}\right)=\int_{\Omega} g_{\epsilon}(x)
$$

onde

$$
g_{\epsilon}(x)= \begin{cases}g(x), & \text { se } x \in \Omega \backslash \omega_{\epsilon} \\ \gamma g(x), & \text { se } x \in \omega_{\epsilon}\end{cases}
$$

A Eq. (2.3.7) pode ser reescrita como:

$$
\psi\left(\chi_{\epsilon}\right)=\int_{\Omega \backslash w_{\epsilon}} g(x)+\int_{w_{\epsilon}} \gamma g(x)
$$

Assim como no primeiro exemplo, soma-se e subtrai-se o valor relacionado ao tamanho da perturbação para se obter o domínio contínuo, de tal forma que:

$$
\psi\left(\chi_{\epsilon}\right)=\int_{\Omega \backslash w_{\epsilon}} g(x)+\int_{w_{\epsilon}} \gamma g(x)+\int_{w_{\epsilon}} g(x)-\int_{w_{\epsilon}} g(x) .
$$


Assim:

$$
\begin{gathered}
\psi\left(\chi_{\epsilon}\right)=\int_{\Omega} g(x)-(1-\gamma) \int_{w_{\epsilon}} g(x) \\
\psi\left(\chi_{\epsilon}\right)=\int_{\Omega} g(x)-(1-\gamma)\left|w_{\epsilon}\right| g(\hat{x})
\end{gathered}
$$

Ou seja, comparando-se com a expansão assintótica topológica da Eq. (2.1.1), conclui-se que a Derivada Topológica é dada por $\Gamma=-(1-\gamma) g(\hat{x})$.

\section{- Exemplo 3}

Este exemplo corresponde à solução de um problema governado por uma EDO de segunda ordem:

$$
\left\{\begin{array}{l}
u_{\epsilon}^{\prime \prime}=0, \\
u_{\epsilon}(0)=0 \\
u_{\epsilon}^{\prime}(1)=1 \\
u_{\epsilon}\left(\epsilon^{+}\right)=u_{\epsilon}\left(\epsilon^{-}\right) \\
u_{\epsilon}^{\prime}\left(\epsilon^{+}\right)=\gamma u_{\epsilon}^{\prime}\left(\epsilon^{-}\right)
\end{array}\right.
$$

A expansão assintótica topológica pode ser obtida ao se substituir os valores de $u_{\epsilon}$ em um funcional. Para se calcular a solução exata, divide-se o problema em 2 partes, referentes à região antes e depois da perturbação. Tem-se, portanto:

$$
\left\{\begin{array}{l}
u_{\epsilon}^{\prime \prime}=0, \\
u_{\epsilon}(0)=0 \\
u_{\epsilon}\left(\epsilon^{+}\right)=u_{\epsilon}\left(\epsilon^{-}\right)
\end{array}\right.
$$

e:

$$
\begin{cases}u_{\epsilon}^{\prime \prime}=0, & \text { em } \epsilon<x<1 \\ u_{\epsilon}^{\prime}(1)=1 & \\ u_{\epsilon}^{\prime}\left(\epsilon^{+}\right)=\gamma u_{\epsilon}^{\prime}\left(\epsilon^{-}\right) & \end{cases}
$$

Integrando-se duas vezes a equação diferencial, obtém-se o resultado da forma $u_{\epsilon}=A x+B$ para antes da perturbação e $u_{\epsilon}=C x+D$ para depois da perturbação, onde $A, B, C$ e $D$ são constantes a serem determinadas. Com a condição de contorno $u_{\epsilon}(0)=0$ calcula-se $B=0$ e $u_{\epsilon}^{\prime}(1)=1, C=1$. Com as condições de contorno nas fronteiras da perturbação tem-se $A=\frac{1}{\gamma} \mathrm{e}$ $D=\frac{\epsilon(1-\gamma)}{\gamma}$. Assim, a solução exata é dada por: 


$$
\left\{\begin{array}{ll}
u_{\epsilon}(x)=\frac{x}{\gamma}, & \text { em } 0<x<\epsilon \\
u_{\epsilon}(x)=x+\epsilon \frac{(1-\gamma)}{\epsilon}, & \text { em } \epsilon<x<1
\end{array} .\right.
$$

Para o domínio contínuo, basta não considerar as condições da perturbação e resolver a equação diferencial, obtendo $u(x)=x$. Assim como no Exemplo 2, $\gamma$ pode ser considerado como uma constante que representa o contraste na propriedade do material, sendo incluído na perturbação.

Os resultados obtidos nas Eqs. (2.3.16), são aplicados no funcional para se determinar a função da derivada topológica a partir da expansão assintótica topológica. Considerando que $u_{\epsilon}(x)$ seja a solução da equação diferencial que minimiza $\psi\left(\chi_{\epsilon}\right)$, a Derivada Topológica é obtida da seguinte forma:

$$
\psi\left(\chi_{\epsilon}\right)=\int_{0}^{1}\left(u_{\epsilon}^{\prime}(x)\right)^{2} d x
$$

O domínio da integral de $\psi\left(\chi_{\epsilon}\right)$ pode ser dividido em duas partes, $0<x<\epsilon$ e $\epsilon<x<1$, e, a partir da condição de contorno $u_{\epsilon}^{\prime}\left(\epsilon^{+}\right)=\gamma u_{\epsilon}^{\prime}\left(\epsilon^{-}\right)$da Eq. (2.3.16), deve-se observar que no primeiro intervalo há um $\gamma$ multiplicando a equação de forma que:

$$
\psi\left(\chi_{\epsilon}\right)=\int_{0}^{\epsilon} \gamma\left(u_{\epsilon}^{\prime}(x)\right)^{2} d x+\int_{\epsilon}^{1}\left(u_{\epsilon}^{\prime}(x)\right)^{2} d x
$$

Como o valor de cada equação já foi calculado e representado na Eq. (2.3.16), chega-se a:

$$
\begin{gathered}
\psi\left(\chi_{\epsilon}\right)=\int_{0}^{\epsilon} \frac{1}{\gamma} d x+\int_{\epsilon}^{1} d x \\
\psi\left(\chi_{\epsilon}\right)=\frac{\epsilon}{\gamma}+1-\epsilon=1+\frac{\epsilon}{\gamma}(1-\gamma) .
\end{gathered}
$$

Para o caso contínuo contínuo, tem-se:

$$
\psi(\chi)=\int_{0}^{1} d x=1
$$

Ou seja, da Eq. (2.3.20) já é possível escrever o problema na forma da expansão assintótica topológica, i.e.:

$$
\psi\left(\chi_{\epsilon}\right)=\psi(\chi)+\frac{\epsilon}{\gamma}(1-\gamma)
$$

Assim, a derivada topológica é expressa como $\Gamma=\frac{1-\gamma}{\gamma}$. Este exemplo se aproxima de uma certa forma do problema de elasticidade linear, pois neste 
caso o que está sendo resolvido é um Laplaciano em um domínio 1D, e, na elasticidade, é buscada uma solução para um Laplaciano em um domínio 2D. Com os conceitos de derivada topológica e expansão assintótica topológica, qualquer problema que envolva o cálculo de um funcional perturbado, seja em um domínio 1D, 2D ou 3D, pode ser resolvido. Vários estudos desta teoria vêm sendo realizados a respeito de sua aplicação no problema de elasticidade $[15,16,17,18]$, por isso, assim como a solução de um Laplaciano em domínio 1D foi obtida, a formulação para um domínio 2D pode ser feita seguindo o mesmo raciocínio. No capítulo seguinte, será apresentado o problema de elasticidade, que resultará na equação da derivada topológica que será aplicada porsteriormente no código PolyTop [19] para se realizar a otimização topológica. 


\section{Cálculo da derivada topológica para o problema de elastici- dade}

Neste capítulo, a partir da definição do problema de elasticidade linear, será desenvolvida a derivada topológica para este caso, segundo Novotny [12]. A função objetivo obtida no final deste capítulo é uma aproximação da energia interna do sistema, que posteriormente, no capítulo 4, será o parâmetro a ser otimizado para que a otimização topológica seja realizada.

\section{1}

\section{Formulação}

A Figura 3.1 ilustra o problema de elasticidade linear bidimensional no estado plano de tensões definidas pelas Eqs. (3.1.1) e (3.1.2),

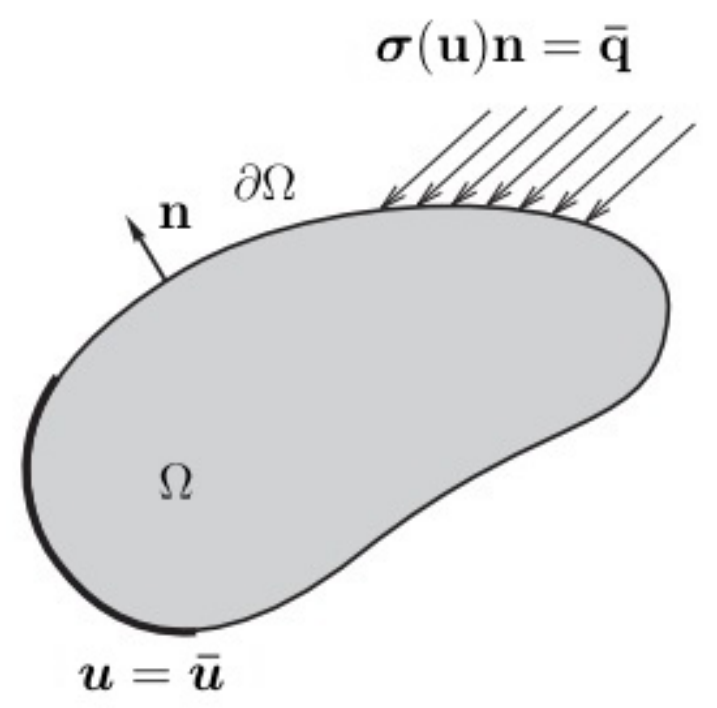

Figura 3.1: Problema de elasticidade: Estado plano de tensões segundo Novotny [8]

A fronteira do domínio $\partial \Omega$ esta sujeita a dois tipos de condições: forças atuantes no sistema $\overline{\boldsymbol{q}}$, denominada condição de Neumann $\Gamma_{N}$ e deformações nas demais regiões chamada de condição de Dirichlet $\Gamma_{D}$. Para resolver este problema, é necessário determinar os deslocamentos $\boldsymbol{u}$, para assim poder 
substituí-los no funcional de forma do domínio, representado pela variação de energia potencial do sistema. Matematicamente, tem-se:

$$
\begin{gathered}
\psi(\chi)=\frac{1}{2} \int_{\Omega} \boldsymbol{\sigma}(\boldsymbol{u}) \cdot \nabla \boldsymbol{u}^{s}-\int_{\Gamma_{N}} \boldsymbol{q} \cdot \boldsymbol{u} \\
\begin{cases}\nabla \cdot \boldsymbol{\sigma}(\boldsymbol{u})=\mathbf{0} & \mathrm{em} \Omega \\
\boldsymbol{\sigma}(\boldsymbol{u})=\mathbb{C} \nabla \boldsymbol{u}^{s} & \\
\boldsymbol{u}=\overline{\boldsymbol{u}} & \mathrm{em} \Gamma_{D} \\
\boldsymbol{\sigma}(\mathbf{u}) \mathbf{n}=\overline{\mathbf{q}} & \mathrm{em} \Gamma_{N} .\end{cases}
\end{gathered}
$$

o termo $\nabla \cdot \boldsymbol{\sigma}(\boldsymbol{u})$ refere-se ao divergente das tensões na forma matricial avaliadas pelas deformações do domínio, $\boldsymbol{n}$ o vetor unitário normal a fronteira $\partial \Omega$. $\nabla \boldsymbol{u}^{s}$ é o tensor de Cauchy dado por:

$$
\nabla \boldsymbol{u}^{s}=\frac{1}{2}\left(\nabla \mathbf{u}+\nabla^{T} \mathbf{u}\right)
$$

Substituido-se a segunda equação da Eq. (3.1.2) na primeira, encontra-se $\Delta \boldsymbol{u}=\mathbf{0}$, isto é, um problema que envolve o Laplaciano das deformações do domínio submetido as duas condições de contorno dadas pelas equações três e quatro da Eq. (3.1.2), onde $\mathbb{C}$ é o tensor constitutivo dado por:

$$
\mathbb{C}=2 \mu \mathbb{\rrbracket}+\lambda \boldsymbol{I} \otimes \boldsymbol{I}
$$

onde $\boldsymbol{I}$ e \ são os tensores identidade de segunda e quarta ordens respectivamente, $\mu$ e $\lambda$ os coeficientes de Lamé, constantes em todo o domínio, e para o estado plano de tensões e de deformações tem-se, respectivamente:

$$
\begin{gathered}
\mu=\frac{E}{2(1+\nu)} \quad \text { e } \lambda=\frac{\nu E}{1-\nu^{2}} \\
\mu=\frac{E}{2(1+\nu)} \quad \text { e } \lambda=\frac{\nu E}{(1+\nu)(1-2 \nu)} .
\end{gathered}
$$

É necessário encontrar a solução do sistema de equações nos domínios perturbado e contínuo. Primeiramente será estudado o domínio contínuo. Para facilitar os cálculos e permitir simplificações posteriores, o problema será transformado da forma diferencial para a integral. Para tanto, multiplicam-se ambos os lados da primeira equação da Eq. (3.1.2) por uma função vetorial $\boldsymbol{\eta}$, que apresenta valor zero para a condição de fronteira de Dirichlet, ou seja, um espaço livre da fronteira do furo onde tem-se apenas a condição de Neumann: 
Capítulo 3. Cálculo da derivada topológica para o problema de elasticidade 31

$$
\int_{\Omega} \nabla \cdot \boldsymbol{\sigma}(\boldsymbol{u}) \boldsymbol{\eta}=0
$$

Pode-se expandir a Eq. (3.1.7) por meio da identidade vetorial de um escalar vezes um vetor, escrita em termos das variáveis em questão, como:

$$
\boldsymbol{\eta} \nabla \cdot \boldsymbol{\sigma}(\boldsymbol{u})=\nabla \cdot[\boldsymbol{\sigma}(\boldsymbol{u}) \boldsymbol{\eta}]-\boldsymbol{\sigma}(\boldsymbol{u}) \nabla \boldsymbol{\eta}
$$

Substituindo-se a Eq. (3.1.8) na Eq. (3.1.7), tem-se:

$$
\int_{\Omega}[\nabla \cdot[\boldsymbol{\sigma}(\boldsymbol{u}) \boldsymbol{\eta}]-\boldsymbol{\sigma}(\boldsymbol{u}) \nabla \boldsymbol{\eta}]=0
$$

A Eq. (3.1.9) ainda pode ser simplificada usando o teorema da divergência, o que gera o seguinte conjunto de equações:

$$
\left\{\begin{array}{l}
\int_{\Omega} \nabla \cdot \boldsymbol{F}=\int_{\partial \Omega} \boldsymbol{F} \cdot \boldsymbol{n} \\
\boldsymbol{F}=\boldsymbol{\eta} \boldsymbol{\sigma}(\boldsymbol{u})
\end{array}\right.
$$

onde $\boldsymbol{F}$ é um campo vetorial contínuo e diferenciável definido na vizinhança de $\Omega$ e $\nabla \cdot \boldsymbol{F}$, o divergente deste campo vetorial. Substituindo-se $\boldsymbol{F}$ na integral do domínio da Eq. (3.1.10), obtém-se:

$$
\int_{\Omega} \nabla \cdot[\boldsymbol{\eta} \boldsymbol{\sigma}(\boldsymbol{u})]=\int_{\partial \Omega} \boldsymbol{\eta} \boldsymbol{\sigma}(\boldsymbol{u}) \cdot \boldsymbol{n}
$$

Aplicando-se a Eq. (3.1.11) na Eq. (3.1.9), chega-se a:

$$
\int_{\Omega} \boldsymbol{\sigma}(\boldsymbol{u}) \cdot \nabla \boldsymbol{\eta}^{s}=\int_{\partial \Omega} \boldsymbol{\eta} \boldsymbol{\sigma}(\boldsymbol{u}) \cdot \boldsymbol{n}
$$

A tensão multiplicada pelo vetor normal é igual às forças que atuam na região, isto é, $\overline{\boldsymbol{q}}=\boldsymbol{n} \boldsymbol{\sigma}(\boldsymbol{u})$ (condição de Neumann) e a integral do contorno do domínio pode ser dividida em duas: Dirichlet e Neumann, ou seja, $\partial \Omega=\Gamma_{N}+\Gamma_{D}$, ambas da Eq. (2.1.2). Substituindo-se essas informações na Eq. (3.1.12), obtém-se:

$$
\int_{\Omega} \boldsymbol{\sigma}(\boldsymbol{u}) \cdot \nabla \boldsymbol{\eta}^{s}=\int_{\Gamma_{D}} \boldsymbol{\eta} \boldsymbol{\sigma}(\boldsymbol{u}) \cdot \boldsymbol{n}+\int_{\Gamma_{N}} \boldsymbol{q} \cdot \boldsymbol{\eta}
$$

Como $\boldsymbol{\eta}$ é zero nas condições de Dirichlet:

$$
\int_{\Omega} \boldsymbol{\sigma}(\boldsymbol{u}) \cdot \nabla \boldsymbol{\eta}^{s}=\int_{\Gamma_{N}} \boldsymbol{q} \cdot \boldsymbol{\eta}
$$


Desta forma, passa-se de um problema diferencial para um problema integral, dado por:

$$
\left\{\begin{array}{l}
\int_{\Omega} \boldsymbol{\sigma}(\boldsymbol{u}) \cdot \nabla \boldsymbol{\eta}^{s}=\int_{\Gamma_{N}} \boldsymbol{q} \cdot \boldsymbol{\eta} \\
\boldsymbol{\sigma}(\boldsymbol{u})=\mathbb{C} \nabla \boldsymbol{u}^{s}
\end{array}\right.
$$

Para o caso em que se tem o domínio perturbado, i.e., com o furo, o funcional de forma pode ser escrito como:

$$
\psi\left(\chi_{\epsilon}\right)=\frac{1}{2} \int_{\Omega} \boldsymbol{\sigma}_{\boldsymbol{\epsilon}}\left(\boldsymbol{u}_{\boldsymbol{\epsilon}}\right) \cdot \nabla \boldsymbol{u}_{\boldsymbol{\epsilon}}^{s}-\int_{\Gamma_{N}} \boldsymbol{q} \cdot \boldsymbol{u}_{\boldsymbol{\epsilon}} .
$$

O sistema associado que resultará na solução de $\boldsymbol{u}_{\epsilon}$ é dado por:

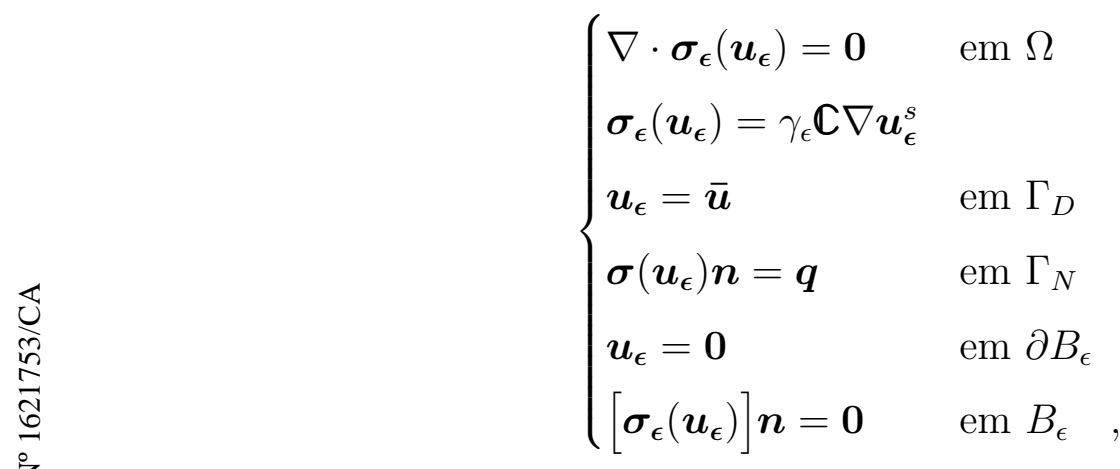

onde $\nabla \cdot \boldsymbol{\sigma}_{\boldsymbol{\epsilon}}\left(\boldsymbol{u}_{\boldsymbol{\epsilon}}\right)$ é o divergente de tensões do domínio perturbado, $\nabla \boldsymbol{u}_{\boldsymbol{\epsilon}}^{s}$ o tensor de Cauchy no domínio perturbado e $\gamma_{\epsilon}$ é a propriedade do material caracterizado pela seguinte função:

$$
\gamma_{\epsilon}=\gamma_{\epsilon}(x)=\left\{\begin{array}{ccc}
1 & \text { se } & x \in \Omega \backslash B_{\epsilon} \\
\gamma & \text { se } & B_{\epsilon},
\end{array}\right.
$$

ou seja, caso a função esteja dentro da região que não há o furo $\Omega \backslash B_{\epsilon}$ (Figura 2.1) o valor é 1, i.e. a densidade do material possui valor máximo. Quando a função encontra-se na região dentro do furo $B_{\epsilon}$ (Figura 2.1) ela terá valor $\gamma$, que varia de $0<\gamma<1$.

Da mesma maneira que no caso do domínio não perturbado, o raciocínio pode ser seguido para se encontrar a forma integral do sistema de equações de $\boldsymbol{u}_{\epsilon}$. Assim, tem-se:

$$
\left\{\begin{array}{l}
\int_{\Omega} \boldsymbol{\sigma}_{\boldsymbol{\epsilon}}\left(\boldsymbol{u}_{\boldsymbol{\epsilon}}\right) \cdot \nabla \boldsymbol{\eta}^{s}=\int_{\Gamma_{N}} \boldsymbol{q} \boldsymbol{\eta} \\
\boldsymbol{\sigma}_{\boldsymbol{\epsilon}}\left(\boldsymbol{u}_{\boldsymbol{\epsilon}}\right)=\gamma_{\epsilon} \mathbb{C} \nabla \boldsymbol{u}_{\boldsymbol{\epsilon}}^{s}
\end{array}\right.
$$


Capítulo 3. Cálculo da derivada topológica para o problema de elasticidade 33

\section{2}

\section{Análise Assintótica}

Para resolver este problema é necessário, primeiramente, calcular $\boldsymbol{u}$ e $\boldsymbol{u}_{\boldsymbol{\epsilon}}$. A aproximação que permitirá o cálculo de $\boldsymbol{u}_{\boldsymbol{\epsilon}}$ pode ser dada por:

$$
u_{\epsilon}(x)=u(x)+w_{\epsilon}(x)+\tilde{u_{\epsilon}}(x)
$$

Esta aproximação pode ser vista no trabalho de Kozlov [20]. Os valores das tensões avaliadas em $\boldsymbol{u}(\boldsymbol{\sigma}(\boldsymbol{u}))$ são facilmente calculadas e as tensões avaliadas em $\boldsymbol{w}_{\boldsymbol{\epsilon}}\left(\boldsymbol{\sigma}\left(\boldsymbol{w}_{\boldsymbol{\epsilon}}\right)\right)$ já existem na literatura, portanto não serão obtidos os valores das deformações, e sim os das tensões avaliadas para cada ponto de interesse. O termo $\tilde{\boldsymbol{u}}_{\boldsymbol{\epsilon}}(x)$ pode ser desprezado, pois seu valor é tão pequeno que não terá impacto no resultado.

Transforma-se a diferença dos funcionais dados pelas Eqs. (3.1.1) e (3.1.16) em uma função apenas definida pelo furo $B_{\epsilon}$. Voltando-se à Eq. (3.1.15) que resulta no valor de $\boldsymbol{u}$ e fazendo $\boldsymbol{\eta}=\boldsymbol{u}_{\boldsymbol{\epsilon}}-\boldsymbol{u}$ [12], tem-se:

$$
\int_{\Omega} \boldsymbol{\sigma}(\boldsymbol{u}) \cdot \nabla\left(\boldsymbol{u}_{\epsilon}-\boldsymbol{u}\right)^{s}=\int_{\Gamma_{N}} \boldsymbol{q} \cdot\left(\boldsymbol{u}_{\epsilon}-\boldsymbol{u}\right)
$$

Separando-se os termos $\boldsymbol{u}$ e $\boldsymbol{u}_{\epsilon}$, e multiplicando-se ambos os lados por 1/2, obtém-se:

$$
\frac{1}{2} \int_{\Omega} \boldsymbol{\sigma}(\boldsymbol{u}) \cdot \nabla \boldsymbol{u}^{s}=\frac{1}{2} \int_{\Omega} \boldsymbol{\sigma}(\boldsymbol{u}) \cdot \nabla \boldsymbol{u}_{\epsilon}^{s}-\frac{1}{2} \int_{\Gamma_{N}} \boldsymbol{q} \cdot\left(\boldsymbol{u}_{\boldsymbol{\epsilon}}-\boldsymbol{u}\right)
$$

Comparando-se a Eq. (3.2.3) com a Eq. (3.1.1) chega-se a:

$$
\psi(\chi)=\frac{1}{2} \int_{\Omega} \boldsymbol{\sigma}(\boldsymbol{u}) \cdot \nabla \boldsymbol{u}_{\epsilon}^{s}-\frac{1}{2} \int_{\Gamma_{N}} \boldsymbol{q} \cdot\left(\boldsymbol{u}_{\boldsymbol{\epsilon}}-\boldsymbol{u}\right)-\int_{\Gamma_{N}} \boldsymbol{q} \cdot \boldsymbol{u}
$$

ou ainda:

$$
\psi(\chi)=\frac{1}{2} \int_{\Omega} \boldsymbol{\sigma}(\boldsymbol{u}) \cdot \nabla \boldsymbol{u}_{\epsilon}^{s}-\frac{1}{2} \int_{\Gamma_{N}} \boldsymbol{q} \cdot\left(\boldsymbol{u}_{\boldsymbol{\epsilon}}+\boldsymbol{u}\right)
$$

Como o material possui propriedades lineares, a Eq. (3.1.15) pode ser reescrita como:

$$
\boldsymbol{\sigma}(\boldsymbol{u}) \cdot \nabla \boldsymbol{u}_{\boldsymbol{\epsilon}}^{s}=\mathbb{C} \nabla \boldsymbol{u}^{s} \cdot \nabla \boldsymbol{u}_{\boldsymbol{\epsilon}}^{s}=\mathbb{C} \nabla \boldsymbol{u}_{\boldsymbol{\epsilon}}^{s} \cdot \nabla \boldsymbol{u}^{s}=\boldsymbol{\sigma}\left(\boldsymbol{u}_{\boldsymbol{\epsilon}}\right) \cdot \nabla \boldsymbol{u}^{s}
$$


Capítulo 3. Cálculo da derivada topológica para o problema de elasticidade 34

Fazendo-se esta mudança na Eq. (3.2.5), tem-se:

$$
\psi(\chi)=\frac{1}{2} \int_{\Omega} \boldsymbol{\sigma}\left(\boldsymbol{u}_{\boldsymbol{\epsilon}}\right) \cdot \nabla \boldsymbol{u}^{s}-\frac{1}{2} \int_{\Gamma_{N}} \boldsymbol{q} \cdot\left(\boldsymbol{u}_{\boldsymbol{\epsilon}}+\boldsymbol{u}\right) .
$$

O funcional do domínio contínuo agora é dado por $\boldsymbol{\sigma}_{\boldsymbol{\epsilon}}\left(\boldsymbol{u}_{\boldsymbol{\epsilon}}\right)$ e o mesmo passo a passo pode ser feito para o domínio perturbado. Com isso, obtém-se:

$$
\psi\left(\chi_{\epsilon}\right)=\frac{1}{2} \int_{\Omega} \boldsymbol{\sigma}_{\boldsymbol{\epsilon}}\left(\boldsymbol{u}_{\epsilon}\right) \cdot \nabla \boldsymbol{u}^{s}-\frac{1}{2} \int_{\Gamma_{N}} \boldsymbol{q} \cdot\left(\boldsymbol{u}_{\boldsymbol{\epsilon}}+\boldsymbol{u}\right)
$$

Desta forma, a variação de energia do sistema é dada por:

$$
\psi\left(\chi_{\epsilon}\right)-\psi(\chi)=\frac{1}{2} \int_{\Omega} \boldsymbol{\sigma}_{\epsilon}\left(\boldsymbol{u}_{\epsilon}\right) \cdot \nabla \boldsymbol{u}^{s}-\frac{1}{2} \int_{\Omega} \boldsymbol{\sigma}\left(\boldsymbol{u}_{\epsilon}\right) \cdot \nabla \boldsymbol{u}^{s}
$$

Voltando-se ao segundo exemplo apresentado no capítulo 2, a integral do domínio $\Omega$ pode ser dividida entre o domínio sem os furos $\Omega \backslash B_{\epsilon}$ e o domínio correspondente aos furos $B_{\epsilon}$. Além disso, quando se trabalha no domínio perturbado, os furos possuem um valor $\gamma \neq 0$, e no domínio contínuo $\gamma=0$, ou seja:

$$
\begin{aligned}
& \psi(\chi)=\frac{1}{2} \int_{\Omega} \boldsymbol{\sigma}\left(\boldsymbol{u}_{\boldsymbol{\epsilon}}\right) \cdot \nabla \boldsymbol{u}^{s}=\frac{1}{2} \int_{\Omega \backslash B_{\epsilon}} \boldsymbol{\sigma}\left(\boldsymbol{u}_{\boldsymbol{\epsilon}}\right) \cdot \nabla \boldsymbol{u}^{s}+\frac{1}{2} \int_{B_{\epsilon}} \boldsymbol{\sigma}\left(\boldsymbol{u}_{\boldsymbol{\epsilon}}\right) \cdot \nabla \boldsymbol{u}^{s} \\
& \psi\left(\chi_{\epsilon}\right)=\frac{1}{2} \int_{\Omega} \boldsymbol{\sigma}_{\boldsymbol{\epsilon}}\left(\boldsymbol{u}_{\boldsymbol{\epsilon}}\right) \cdot \nabla \boldsymbol{u}^{s}=\frac{1}{2} \int_{\Omega \backslash B_{\epsilon}} \boldsymbol{\sigma}\left(\boldsymbol{u}_{\boldsymbol{\epsilon}}\right) \cdot \nabla \boldsymbol{u}^{s}+\frac{1}{2} \int_{B_{\epsilon}} \gamma \boldsymbol{\sigma}\left(\boldsymbol{u}_{\boldsymbol{\epsilon}}\right) \cdot \nabla \boldsymbol{u}^{s}
\end{aligned}
$$

Assim, ao se subtrair a Eq. (3.2.11) da Eq. (3.2.10), ou seja, fazer $\psi\left(\chi_{\epsilon}\right)-\psi(\chi)$, o termo que se refere a região sem o furo desaparece, restando apenas o furo $B_{\epsilon}$, ou seja:

$$
\psi\left(\chi_{\epsilon}\right)-\psi(\chi)=-\frac{1-\gamma}{2} \int_{B_{\epsilon}} \boldsymbol{\sigma}\left(\boldsymbol{u}_{\epsilon}\right) \cdot \nabla \boldsymbol{u}^{s}
$$

Ou, em termos de $\boldsymbol{\sigma}_{\epsilon}$ :

$$
\psi\left(\chi_{\epsilon}\right)-\psi(\chi)=-\frac{1-\gamma}{2 \gamma} \int_{B_{\epsilon}} \boldsymbol{\sigma}_{\epsilon}\left(\boldsymbol{u}_{\epsilon}\right) \cdot \nabla \boldsymbol{u}^{s} .
$$

Portanto, a Derivada Topológica depende apenas de $\boldsymbol{\sigma}_{\epsilon}\left(\boldsymbol{u}_{\epsilon}\right)$, ou seja, é necessário calcular $\boldsymbol{\sigma}_{\boldsymbol{\epsilon}}(\boldsymbol{u})$ e $\boldsymbol{\sigma}_{\boldsymbol{\epsilon}}\left(\boldsymbol{w}_{\boldsymbol{\epsilon}}\right)$ para se obter a expressão desejada. 
Capítulo 3. Cálculo da derivada topológica para o problema de elasticidade 35

\section{3}

\section{Cálculo da tensão}

Como a diferença entre os funcionais $\psi\left(\chi_{\epsilon}\right)-\psi(\chi)$ depende exclusivamente da tensão gerada pelo furo no domínio perturbado, aplica-se o operador $\boldsymbol{\sigma}_{\boldsymbol{\epsilon}}$, que representa as tensões no domínio perturbado, à Eq. (3.2.1) de tal forma que é obtido:

$$
\sigma_{\epsilon}\left(u_{\epsilon}(x)\right)=\sigma_{\epsilon}(u(x))+\sigma_{\epsilon}\left(w_{\epsilon}(x)\right)+\sigma_{\epsilon}\left(\tilde{u}_{\epsilon}(x)\right)
$$

Os deslocamentos no domínio não perturbado $\boldsymbol{u}(\boldsymbol{x})$ podem ser expandidos usando o teorema do valor médio, i.e.:

$$
\boldsymbol{\sigma}_{\boldsymbol{\epsilon}}\left(\boldsymbol{u}_{\boldsymbol{\epsilon}}(\boldsymbol{x})\right)=\boldsymbol{\sigma}_{\boldsymbol{\epsilon}}(\boldsymbol{u}(\hat{\boldsymbol{x}}))+\gamma \nabla \boldsymbol{\sigma}(\boldsymbol{u}(\boldsymbol{y}))(\boldsymbol{x}-\hat{\boldsymbol{x}})+\boldsymbol{\sigma}_{\boldsymbol{\epsilon}}\left(\boldsymbol{w}_{\boldsymbol{\epsilon}}(\boldsymbol{x})\right)+\boldsymbol{\sigma}_{\boldsymbol{\epsilon}}\left(\tilde{\boldsymbol{u}}_{\boldsymbol{\epsilon}}(\boldsymbol{x})\right)
$$

O termo $\gamma \boldsymbol{\sigma}(\boldsymbol{u}(\boldsymbol{y}))$ aparece devido à igualdade $\boldsymbol{\sigma}_{\boldsymbol{\epsilon}}(\boldsymbol{u}(\boldsymbol{y}))=\gamma \boldsymbol{\sigma}(\boldsymbol{u}(\boldsymbol{y}))$, onde $y$ corresponde ao ponto intermediário entre $\boldsymbol{x}$ e $\hat{\boldsymbol{x}}$, ou seja, entre um ponto no contorno do furo $\partial B_{\epsilon}$ e o ponto central do furo. Das equações relacionadas com o sistema do domínio perturbado sabe-se que a tensão $\boldsymbol{\sigma}_{\boldsymbol{\epsilon}}\left(\boldsymbol{u}_{\boldsymbol{\epsilon}}(\boldsymbol{x})\right)$, no contorno do furo, pode ser dada por $\left[\boldsymbol{\sigma}_{\epsilon}\left(\boldsymbol{u}_{\epsilon}\right)\right] \boldsymbol{n}=\mathbf{0}$, chegando-se a:

$$
\left(\boldsymbol{\sigma}\left(\boldsymbol{u}_{\epsilon}\right)_{\Omega \backslash B_{\epsilon}}-\gamma \boldsymbol{\sigma}\left(\boldsymbol{u}_{\epsilon}\right)_{B_{\epsilon}}\right) \boldsymbol{n}+\left[\boldsymbol{\sigma}_{\boldsymbol{\epsilon}}\left(\boldsymbol{w}_{\boldsymbol{\epsilon}}(\boldsymbol{x})\right)\right] \boldsymbol{n}+\left[\boldsymbol{\sigma}_{\boldsymbol{\epsilon}}\left(\tilde{\boldsymbol{u}}_{\boldsymbol{\epsilon}}(\boldsymbol{x})\right)\right] \boldsymbol{n}=\mathbf{0}
$$

Substituindo-se a Eq. (3.3.1) na Eq. (3.3.3), chega-se a:

$$
\begin{array}{r}
{\left[\boldsymbol{\sigma}(\boldsymbol{u}(\hat{\boldsymbol{x}}))+\nabla \boldsymbol{\sigma}(\boldsymbol{u}(\boldsymbol{y}))(\boldsymbol{x}-\hat{\boldsymbol{x}})+\boldsymbol{\sigma}\left(\boldsymbol{w}_{\boldsymbol{\epsilon}}(\boldsymbol{x})\right)-\gamma \boldsymbol{\sigma}(\boldsymbol{u}(\hat{\boldsymbol{x}})-\right.} \\
\left.-\gamma \nabla \boldsymbol{\sigma}(\boldsymbol{u}(\boldsymbol{y}))(\boldsymbol{x}-\hat{\boldsymbol{x}})-\gamma \boldsymbol{\sigma}\left(\boldsymbol{w}_{\boldsymbol{\epsilon}}(\boldsymbol{x})\right)\right] \boldsymbol{n}+\left[\boldsymbol{\sigma}_{\boldsymbol{\epsilon}}\left(\boldsymbol{w}_{\boldsymbol{\epsilon}}(\boldsymbol{x})\right)\right] \boldsymbol{n}+\left[\boldsymbol{\sigma}_{\boldsymbol{\epsilon}}\left(\tilde{\boldsymbol{u}}_{\boldsymbol{\epsilon}}(\boldsymbol{x})\right)\right] \boldsymbol{n}=\mathbf{0}
\end{array}
$$

A diferença $\boldsymbol{x}-\hat{\boldsymbol{x}}$ é um vetor cujo módulo $\epsilon$ é o raio do furo, e cuja direção é $-\boldsymbol{n}$, ou seja, para dentro do furo. Fazendo-se $\boldsymbol{x}-\hat{\boldsymbol{x}}=-\epsilon \boldsymbol{n}$ e substituindo-se na Eq. (3.3.4), obtém-se:

$$
\begin{array}{r}
{\left[\boldsymbol{\sigma}(\boldsymbol{u}(\hat{\boldsymbol{x}}))-\nabla \boldsymbol{\sigma}(\boldsymbol{u}(\boldsymbol{y}))(\epsilon \boldsymbol{n})+\boldsymbol{\sigma}\left(\boldsymbol{w}_{\boldsymbol{\epsilon}}(\boldsymbol{x})\right)-\gamma \boldsymbol{\sigma}(\boldsymbol{u}(\hat{\boldsymbol{x}})+\gamma \nabla \boldsymbol{\sigma}(\boldsymbol{u}(\boldsymbol{y}))(\epsilon \boldsymbol{n})-\right.} \\
\left.-\gamma \boldsymbol{\sigma}\left(\boldsymbol{w}_{\boldsymbol{\epsilon}}(\boldsymbol{x})\right)\right] \boldsymbol{n}+\left[\boldsymbol{\sigma}_{\boldsymbol{\epsilon}}\left(\boldsymbol{w}_{\boldsymbol{\epsilon}}(\boldsymbol{x})\right)\right] \boldsymbol{n}+\left[\boldsymbol{\sigma}_{\boldsymbol{\epsilon}}\left(\tilde{\boldsymbol{u}}_{\boldsymbol{\epsilon}}(\boldsymbol{x})\right) \boldsymbol{n}=\mathbf{0}\right.
\end{array}
$$


Capítulo 3. Cálculo da derivada topológica para o problema de elasticidade 36

Finalmente, a Eq. (3.3.5), que está avaliada sobre o contorno do furo $\partial B$, pode ser reduzida a:

$$
(1-\gamma) \boldsymbol{\sigma}(\boldsymbol{u}(\hat{\boldsymbol{x}})) \boldsymbol{n}-\epsilon(1-\gamma) \nabla \boldsymbol{\sigma}(\boldsymbol{u}(\boldsymbol{y})) \boldsymbol{n}) \boldsymbol{n}+\left[\boldsymbol{\sigma}_{\epsilon}\left(\boldsymbol{w}_{\epsilon}(\boldsymbol{x})\right)\right] \boldsymbol{n}+\left[\boldsymbol{\sigma}_{\epsilon}\left(\boldsymbol{u}_{\epsilon}(\boldsymbol{x})\right)\right] \boldsymbol{n}=\mathbf{0}
$$

Desta maneira, pode-se escolher $\left[\boldsymbol{\sigma}_{\boldsymbol{\epsilon}}\left(\boldsymbol{w}_{\boldsymbol{\epsilon}}(\boldsymbol{x})\right] \boldsymbol{n}\right.$ e $\left[\boldsymbol{\sigma}_{\boldsymbol{\epsilon}}(\tilde{\boldsymbol{u}}(\boldsymbol{x}))\right] \boldsymbol{n}$ como, respectivamente:

$$
\begin{gathered}
{\left[\boldsymbol{\sigma}_{\epsilon}\left(\boldsymbol{w}_{\boldsymbol{\epsilon}}\right)\right] \boldsymbol{n}=-(1-\gamma) \boldsymbol{\sigma}(\boldsymbol{u}(\hat{\boldsymbol{x}})) \boldsymbol{n}} \\
\left.\left[\boldsymbol{\sigma}_{\boldsymbol{\epsilon}}(\tilde{\boldsymbol{u}}(\boldsymbol{x}))\right] \boldsymbol{n}=-\epsilon(1-\gamma) \nabla \boldsymbol{\sigma}(\boldsymbol{u}(\boldsymbol{y})) \boldsymbol{n}\right) \boldsymbol{n}
\end{gathered}
$$

O valor de $\left[\boldsymbol{\sigma}_{\boldsymbol{\epsilon}}(\tilde{\boldsymbol{u}}(\boldsymbol{x}))\right] \boldsymbol{n}$ não será considerado, pois, como dito anteriormente, é desprezível. Para a Eq. (3.3.3) formula-se o seguinte problema:

$$
\begin{cases}\nabla \cdot \boldsymbol{\sigma}_{\boldsymbol{\epsilon}}\left(\boldsymbol{w}_{\boldsymbol{\epsilon}}\right)=0 & \text { em } \mathbb{R}^{2} \\ \boldsymbol{\sigma}_{\boldsymbol{\epsilon}}\left(\boldsymbol{w}_{\boldsymbol{\epsilon}}\right) \rightarrow 0 & \text { quando } \Omega \rightarrow \infty \\ {\left[\boldsymbol{\sigma}_{\boldsymbol{\epsilon}}\left(\boldsymbol{w}_{\boldsymbol{\epsilon}}\right)\right] \boldsymbol{n}=-(1-\gamma) \boldsymbol{\sigma}(\boldsymbol{u}(\hat{\boldsymbol{x}})) \boldsymbol{n}} & \text { em } \partial B\end{cases}
$$

A solução desse problema, conhecido como teorema de Eshelby [9], pode ser encontrada no livro de Mura [21] e é dada por:

$$
\boldsymbol{\sigma}_{\epsilon}\left(\boldsymbol{w}_{\boldsymbol{\epsilon}}\right)=\mathbb{T}_{\gamma} \boldsymbol{\sigma}(\boldsymbol{u}(\hat{\boldsymbol{x}}))
$$

onde $\mathbb{T}_{\gamma}$ é um tensor uniforme constante de quarta ordem dado por:

$$
\mathbb{T}_{\gamma}=\frac{1}{2} \frac{\gamma(1-\gamma)}{1+\gamma \beta}\left(2 \beta \mathbb{1}+\frac{\alpha-\beta}{1-\gamma \alpha} \boldsymbol{I} \otimes \boldsymbol{I}\right)
$$

com:

$$
\alpha=\frac{\mu+\lambda}{\mu}
$$

e:

$$
\beta=\frac{3 \mu+\lambda}{\mu+\lambda}
$$




\section{4}

\section{Determinação do funcional da energia interna do sistema}

Uma vez calculada a diferença do funcional e realizada a análise assintótica, é possível encontrar a derivada topológica a partir da expansão assintótica topológica. Como a aproximação do resultado encontrado é $\boldsymbol{u}_{\boldsymbol{\epsilon}}=\boldsymbol{u}+\boldsymbol{w}_{\boldsymbol{\epsilon}}$ e a equação da diferença de energia contempla apenas a tensão no domínio não perturbado $\boldsymbol{\sigma}$, é necessário achar uma relação entre $\boldsymbol{\sigma}$ e $\boldsymbol{\sigma}_{\boldsymbol{\epsilon}}$. Para isso, voltandose às Eqs. (3.1.2) e (3.1.17), encontra-se o conjunto de equações:

$$
\left\{\begin{array}{l}
\boldsymbol{\sigma}(\boldsymbol{u})=\mathbb{C} \nabla \boldsymbol{u}^{s} \\
\boldsymbol{\sigma}_{\boldsymbol{\epsilon}}\left(\boldsymbol{u}_{\boldsymbol{\epsilon}}\right)=\gamma \mathbb{C} \nabla \boldsymbol{u}_{\boldsymbol{\epsilon}}^{s}
\end{array}\right.
$$

Da primeira equação, pode-se concluir que $\boldsymbol{\sigma}\left(\boldsymbol{u}_{\epsilon}\right)=\mathbb{C} \nabla \boldsymbol{u}_{\epsilon}^{s}$ e, substituindo-se na segunda, obtém-se:

$$
\boldsymbol{\sigma}\left(\boldsymbol{u}_{\epsilon}\right)=\frac{1}{\gamma} \boldsymbol{\sigma}_{\epsilon}\left(\boldsymbol{u}_{\epsilon}\right)
$$

Desta forma, a expansão de $\boldsymbol{u}_{\boldsymbol{\epsilon}}$, definida pela Eq. (3.2.1) e substituída na Eq. (3.2.12), conduz a:

$$
\psi\left(\chi_{\epsilon}\right)-\psi(\chi)=-\frac{1-\gamma}{2} \int_{B_{\epsilon}} \boldsymbol{\sigma}(\boldsymbol{u}(\boldsymbol{x})) \cdot \nabla \boldsymbol{u}^{s}(\boldsymbol{x})-\frac{1-\gamma}{2 \gamma} \int_{B_{\epsilon}} \boldsymbol{\sigma}_{\boldsymbol{\epsilon}}\left(\boldsymbol{w}_{\boldsymbol{\epsilon}}(\boldsymbol{x})\right) \cdot \nabla \boldsymbol{u}^{s}(\boldsymbol{x})
$$

Voltando-se à equação original da expansão assintótica topológica dada pela Eq. (2.1.1), do lado esquerdo tem-se a diferença dos funcionais e do direito é necessário encontrar uma forma parecida com $\mathcal{T}(\hat{x}) f(\epsilon)$. Trabalhando-se com o primeiro termo da expansão 3.4.3, soma-se e subtrai-se a integral de $\boldsymbol{\sigma}(\hat{\boldsymbol{x}}) \cdot \nabla \boldsymbol{u}(\hat{\boldsymbol{x}})$, tal como nos exemplos 1, 2 e 3 do capítulo 2, chegando-se a:

$$
\begin{aligned}
\int_{B_{\epsilon}} \boldsymbol{\sigma}(\boldsymbol{u}(\boldsymbol{x})) \cdot \nabla \boldsymbol{u}^{s}(\boldsymbol{x})=\int_{B_{\epsilon}} \boldsymbol{\sigma}(\boldsymbol{u}(\boldsymbol{x})) \cdot \nabla \boldsymbol{u}^{s}(\boldsymbol{x})+ & \int_{B_{\epsilon}} \boldsymbol{\sigma}(\boldsymbol{u}(\hat{\boldsymbol{x}})) \cdot \nabla \boldsymbol{u}^{s}(\hat{\boldsymbol{x}})- \\
& -\int_{B_{\epsilon}} \boldsymbol{\sigma}(\boldsymbol{u}(\hat{\boldsymbol{x}})) \cdot \nabla \boldsymbol{u}^{s}(\hat{\boldsymbol{x}})
\end{aligned}
$$

Rearrumando-se os termos:

$$
\begin{array}{r}
\int_{B_{\epsilon}} \boldsymbol{\sigma}(\boldsymbol{u}(\boldsymbol{x})) \cdot \nabla \boldsymbol{u}^{s}(\boldsymbol{x})=\int_{B_{\epsilon}} \boldsymbol{\sigma}(\boldsymbol{u}(\hat{\boldsymbol{x}})) \cdot \nabla \boldsymbol{u}^{s}(\hat{\boldsymbol{x}})+ \\
+\int_{B_{\epsilon}}\left(\boldsymbol{\sigma}(\boldsymbol{u}(\boldsymbol{x})) \cdot \nabla \boldsymbol{u}^{s}-\boldsymbol{\sigma}(\boldsymbol{u}(\hat{\boldsymbol{x}})) \cdot \nabla \boldsymbol{u}^{s}(\hat{\boldsymbol{x}})\right)
\end{array}
$$


Capítulo 3. Cálculo da derivada topológica para o problema de elasticidade 38

Porém, como $\boldsymbol{\sigma}(\boldsymbol{u}(\hat{\boldsymbol{x}})) \cdot \nabla \boldsymbol{u}^{s}(\hat{\boldsymbol{x}})$ é um valor em um ponto, ou seja, um valor constante, pode-se retirá-lo da integral, restando apenas parcelas associadas ao furo. No caso 2D, esta é a área da perturbação, isto é, a área do círculo que corresponde a $\pi \epsilon^{2}$, ou seja,

$$
\begin{aligned}
& \int_{B_{\epsilon}} \boldsymbol{\sigma}(\boldsymbol{u}(\boldsymbol{x})) \cdot \nabla \boldsymbol{u}^{s}(\boldsymbol{x})=\pi \epsilon^{2} \boldsymbol{\sigma}(\boldsymbol{u}(\hat{\boldsymbol{x}})) \cdot \nabla \boldsymbol{u}^{s}(\hat{\boldsymbol{x}})+ \\
& \quad+\int_{B_{\epsilon}}\left(\boldsymbol{\sigma}(\boldsymbol{u}(\boldsymbol{x})) \cdot \nabla \boldsymbol{u}^{s}(\boldsymbol{x})-\boldsymbol{\sigma}(\boldsymbol{u}(\hat{\boldsymbol{x}})) \cdot \nabla \boldsymbol{u}^{s}(\hat{\boldsymbol{x}})\right)
\end{aligned}
$$

A partir da Eq. (3.4.6) pode-se observar que existem dois termos: o de ordem $1\left(\mathrm{o}\left(\epsilon^{1}\right)\right)$ e o de ordem $2\left(\mathrm{o}\left(\epsilon^{2}\right)\right)$. Como a Derivada Topológica, que será obtida neste trabalho, é apenas de ordem 1, pode-se reescrever esta equação como:

$$
\int_{B_{\epsilon}} \boldsymbol{\sigma}(\boldsymbol{u}(\boldsymbol{x})) \cdot \nabla \boldsymbol{u}^{s}(\boldsymbol{x})=\pi \epsilon^{2} \boldsymbol{\sigma}(\boldsymbol{u}(\hat{\boldsymbol{x}})) \cdot \nabla \boldsymbol{u}^{s}(\hat{\boldsymbol{x}})+o\left(\epsilon^{2}\right)
$$

Voltando-se a Eq. (3.4.3), é necessário expandir o termo $\boldsymbol{w}_{\boldsymbol{\epsilon}}$ da mesma forma que $\boldsymbol{u}$, ou seja,

$$
\begin{aligned}
\int_{B_{\epsilon}} \boldsymbol{\sigma}_{\boldsymbol{\epsilon}}\left(\boldsymbol{w}_{\boldsymbol{\epsilon}}(\boldsymbol{x})\right) \cdot \nabla \boldsymbol{u}^{s}(\boldsymbol{x})=\int_{B_{\epsilon}} \boldsymbol{\sigma}_{\boldsymbol{\epsilon}}\left(\boldsymbol{w}_{\boldsymbol{\epsilon}}(\boldsymbol{x})\right) \cdot \nabla \boldsymbol{u}^{s}(\boldsymbol{x})+ & \int_{B_{\epsilon}} \boldsymbol{\sigma}_{\boldsymbol{\epsilon}}\left(\boldsymbol{w}_{\epsilon}(\boldsymbol{x})\right) \cdot \nabla \boldsymbol{u}^{s}(\hat{\boldsymbol{x}})- \\
& -\int_{B_{\epsilon}} \boldsymbol{\sigma}_{\boldsymbol{\epsilon}}\left(\boldsymbol{w}_{\epsilon}(\boldsymbol{x})\right) \cdot \nabla \boldsymbol{u}^{s}(\hat{\boldsymbol{x}})
\end{aligned}
$$

Combinando-se o primeiro e o terceiro termos da Eq. (3.4.8), obtém-se:

$$
\begin{array}{r}
\int_{B_{\epsilon}} \boldsymbol{\sigma}_{\epsilon}\left(\boldsymbol{w}_{\boldsymbol{\epsilon}}(\boldsymbol{x})\right) \cdot \nabla \boldsymbol{u}^{s}(\boldsymbol{x})=\int_{B_{\epsilon}} \boldsymbol{\sigma}_{\boldsymbol{\epsilon}}\left(\boldsymbol{w}_{\boldsymbol{\epsilon}}(\boldsymbol{x})\right) \cdot \nabla \boldsymbol{u}^{s}(\hat{\boldsymbol{x}})+ \\
+\int_{B_{\epsilon}} \boldsymbol{\sigma}_{\epsilon}\left(\boldsymbol{w}_{\boldsymbol{\epsilon}}(\boldsymbol{x})\right) \cdot\left(\nabla \boldsymbol{u}^{s}(\boldsymbol{x})-\nabla \boldsymbol{u}^{s}(\hat{\boldsymbol{x}})\right)
\end{array}
$$

Como $\boldsymbol{\sigma}_{\boldsymbol{\epsilon}}\left(\boldsymbol{w}_{\boldsymbol{\epsilon}}(\boldsymbol{x})\right)$ foi calculado a partir do teorema de Eshelby, pode-se escrever:

$$
\int_{B_{\epsilon}} \boldsymbol{\sigma}_{\epsilon}\left(\boldsymbol{w}_{\epsilon}(\boldsymbol{x})\right) \cdot \nabla \boldsymbol{u}^{s}=\pi \epsilon^{2} \mathbb{T}_{\gamma} \boldsymbol{\sigma}(\boldsymbol{u}(\hat{\boldsymbol{x}})) \cdot \nabla \boldsymbol{u}^{s}(\hat{\boldsymbol{x}})+o\left(\epsilon^{2}\right)
$$


Finalmente, substituindo-se a Eq. (3.4.7) e a Eq. (3.4.10) na Eq. (3.4.3) e, como $\mathbb{T}_{\gamma}$ é um tensor de quarta ordem, tem-se:

$$
\begin{aligned}
\psi\left(\chi_{\epsilon}\right)-\psi(\chi)=- & \frac{1-\gamma}{2}\left(\pi \epsilon^{2} \mathbb{1} \boldsymbol{\sigma}(\boldsymbol{u}(\hat{\boldsymbol{x}})) \cdot \nabla \boldsymbol{u}^{s}(\hat{\boldsymbol{x}})+o\left(\epsilon^{2}\right)\right)- \\
& -\frac{1-\gamma}{2 \gamma}\left(\pi \epsilon^{2} \mathbb{T}_{\gamma} \boldsymbol{\sigma}(\boldsymbol{u}(\hat{\boldsymbol{x}})) \cdot \nabla \boldsymbol{u}^{s}(\hat{\boldsymbol{x}})+o\left(\epsilon^{2}\right)\right)
\end{aligned}
$$

onde $\mathbb{1}$ é o tensor identidade de quarta ordem. De forma simplificada, podemos escrever:

$$
\begin{gathered}
\psi\left(\chi_{\epsilon}\right)-\psi(\chi)=-\pi \epsilon^{2} \frac{1-\gamma}{2 \gamma}\left(\mathbb{T}_{\gamma}+\gamma \mathbb{1}\right) \boldsymbol{\sigma}(\boldsymbol{u}(\hat{\boldsymbol{x}})) \cdot \nabla \boldsymbol{u}^{s}(\hat{\boldsymbol{x}})+o\left(\epsilon^{2}\right) \\
\psi\left(\chi_{\epsilon}\right)-\psi(\chi)=-\pi \epsilon^{2} \mathbb{P}_{\gamma} \boldsymbol{\sigma}(\boldsymbol{u}(\hat{\boldsymbol{x}})) \cdot \nabla \boldsymbol{u}^{s}(\hat{\boldsymbol{x}})+o\left(\epsilon^{2}\right) \\
\mathbb{P}_{\gamma}=-\frac{1-\gamma}{2 \gamma}\left(\mathbb{T}_{\gamma}+\gamma \mathbb{1}\right)
\end{gathered}
$$

A Eq. (3.4.13) corresponde a expansão assintótica topológica, em que a função da perturbação é $f(\epsilon)=\pi \epsilon^{2}$, e a derivada topológica é dada pela Eq. $(3.4 .5)$ :

$$
\Gamma(\hat{x})=-\mathbb{P}_{\gamma} \boldsymbol{\sigma}(\boldsymbol{u}(\hat{\boldsymbol{x}})) \cdot \nabla \boldsymbol{u}^{s}(\hat{\boldsymbol{x}})
$$

Algumas simplificações podem ser feitas na expressão final da derivada topológica. Expandindo-se $\mathbb{P}_{\gamma}$, e em seguida, $\mathbb{T}_{\gamma}$, obtém-se:

$$
\left.\Gamma(\hat{\boldsymbol{x}})=\left[\frac{1}{2} \frac{\gamma(1-\gamma)}{1+\gamma \beta}\left[(1+\beta) \mathbb{1}+\frac{1}{2}(\alpha-\beta) \frac{1-\gamma}{1+\gamma \alpha} \mathbf{I} \otimes \mathbf{I}\right)\right]\right] \boldsymbol{\sigma}(\boldsymbol{u}(\hat{\boldsymbol{x}})) \cdot \nabla \boldsymbol{u}^{s}(\hat{\boldsymbol{x}})
$$

Ao pesquisar os valores para o coeficiente de Poisson de materiais utilizados para a construção de estruturas, observa-se que seus valores possuem um valor próximo de $\nu=\frac{1}{3}$. Este valor permite que a segunda parte da Eq. (3.4.16) seja igual a 0 , pois substituindo os parâmetros $\alpha, \beta, \lambda$ e $\nu$ nesta, encontra-se que a equação no numerador da segunda parte é $N_{2}=2(3 \nu-1)$, ou seja, tornando zero esta parte da equação. Assim, tem-se: 
Capítulo 3. Cálculo da derivada topológica para o problema de elasticidade 40

$$
\Gamma(\hat{x})=-\left[\frac{3}{2} \frac{\gamma-1}{1+2 \gamma}\right] \boldsymbol{\sigma}(\boldsymbol{u}(\hat{\boldsymbol{x}})) \cdot \nabla \boldsymbol{u}^{s}(\hat{\boldsymbol{x}})
$$

Finalmente, segundo Itskov [22], pode-se mostrar que:

$$
\mathbb{1} \boldsymbol{\sigma}(\boldsymbol{u}(\hat{\boldsymbol{x}})) \cdot \nabla \boldsymbol{u}^{s}(\hat{\boldsymbol{x}})=\boldsymbol{\sigma}(\boldsymbol{u}(\hat{\boldsymbol{x}})): \nabla \boldsymbol{u}(\hat{\boldsymbol{x}})^{s}
$$

chegando-se na forma final da derivada topológica:

$$
\Gamma(\hat{\boldsymbol{x}})=-\left[\frac{3}{2} \frac{\gamma-1}{1+2 \gamma}\right] \boldsymbol{\sigma}(\boldsymbol{u}(\hat{\boldsymbol{x}})): \nabla \boldsymbol{u}^{s}(\hat{\boldsymbol{x}})
$$

Considerando $\gamma=0$, a perturbação torna-se um vazio, de forma que a Eq. (3.4.19) torna-se:

$$
\psi(\hat{\boldsymbol{x}})=\psi(\hat{\boldsymbol{x}})-\pi \epsilon^{2}\left[\frac{3}{2} \boldsymbol{\sigma}(\boldsymbol{u}(\hat{\boldsymbol{x}})): \nabla \boldsymbol{u}^{s}(\hat{\boldsymbol{x}})\right] .
$$

Desta forma, o problema de otimização topológica passa a ser minimizar a equação da energia interna do sistema, Eq. (3.4.20), respeitando a condição de equilíbrio $\boldsymbol{K} \boldsymbol{u}=\boldsymbol{q}$. No próximo capítulo serão utilizados estes resultados para implementar-se o algoritmo de otimização. 


\section{4 \\ Implementação da Derivada Topológica}

Neste capítulo, mostraremos primeiramente as vantagens em se utilizar malhas poligonais; em seguida, a equação obtida no capítulo anterior para o problema de elasticidade linear será utilizada para realizar a otimização topológica, pois, através da minimização daquela, a topologia final será encontrada e discutida em 4.2 .

\section{1}

\section{Elementos Quadrilaterais x Elementos Poligonais}

As aplicações de otimização topológica mais conhecidas na literatura técnica apontam para o uso de elementos finitos convencionais (ex: triângulos e quadriláteros) na discretização dos domínios bidimensionais [23,24]. No entanto, mais recentemente, diversos trabalhos de otimização topológica propõem o uso de elementos poligonais [25,26]. Diversos problemas numéricos encontrados com a utilização de elementos convencionais são naturalmente solucionados ao se utilizar elementos poligonais. Dois dos principais problemas encontrados na otimização topológica são discutidos aqui: o problema de checkerboard e o problema de one-node connection.

- Problemas de Checkerboard ou Tabuleiro de Xadrez

Conforme mencionado anteriormente, o uso de elementos finitos de baixa ordem, ou seja, elementos triangulares ou quadrilaterias com variação linear dos deslocamentos e densidade constante (tal como ilustrado na Figura 4.1) podem induzir o problema de checkerboard. 


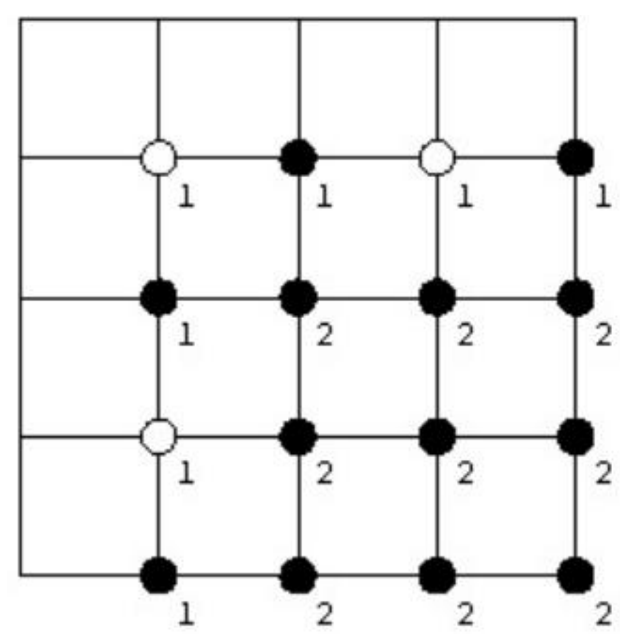

(a)

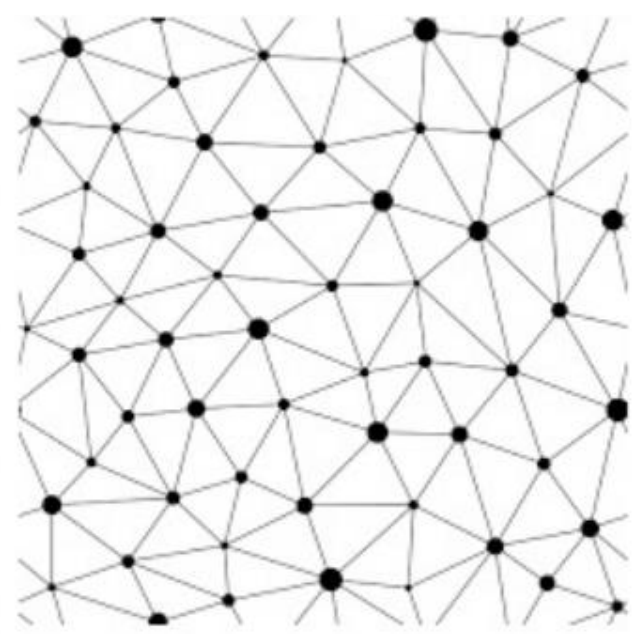

(b)

Figura 4.1: Exemplo de baixa ordem de elementos: (a) quadrilaterias; e (b) triangulares

Talishi et. al. [27] propuseram a utilização de elementos finitos poligonais na solução do problema de otimização topológica em domínios bidimensionais arbitrários (i.e. não cartesianos). O termo checkerboard é utilizado na literatura devido ao aspecto final da solução do problema de otimização topológica se assemelhar a um tabuleiro de xadrez, como ilustrado na Figura 4.2.

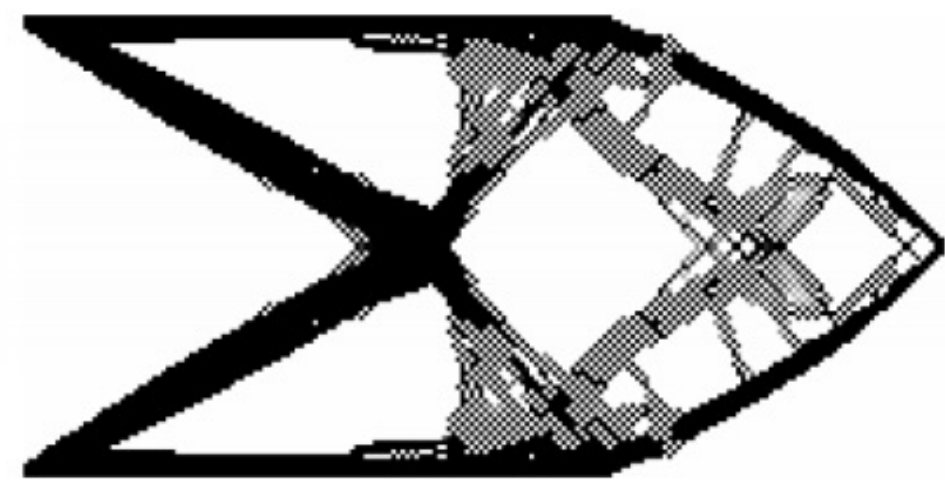

Figura 4.2: Problema de checkerboard; Fonte: [28] 
Trata-se de uma instalibidade numérica provocada pela escolha inadequada dos campos de interpolação dos deslocamentos e densidade. Os elementos de baixa ordem não satisfazem à condição de LBB (LadyzenskajaBabuška-Brezzi) [29] e apresentam instabilidade do tabuleiro de xadrez. Uma das maneiras de resolver este problema é usar um elemento de ordem maior para modelar a estrutura (elementos quadráticos com densidade variando linearmente, por exemplo).

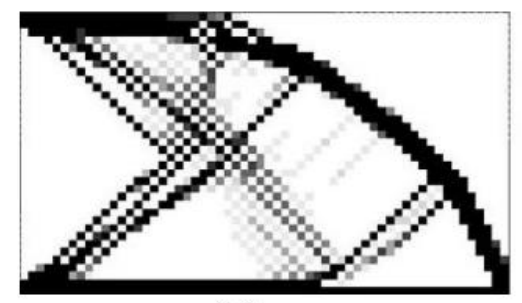

(a)

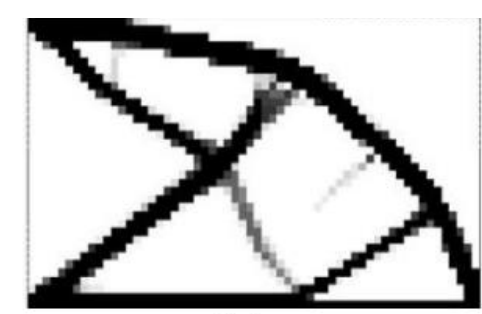

(b)

Figura 4.3: Comparação entre (a) elementos de quatro nós e (b) elementos de oito nós; Fonte: [30]

A Figura 4.3 ilustra uma das formas de se eliminar o problema de checkerboard por meio do aumento da ordem de interpolação dos campos de deslocamentos e densidades (ver Shuckla [30]). Neste trabalho será utilizado o programa PolyTop [19] que possui um código capaz de gerar malhas poligonais como as da Figura 4.4:

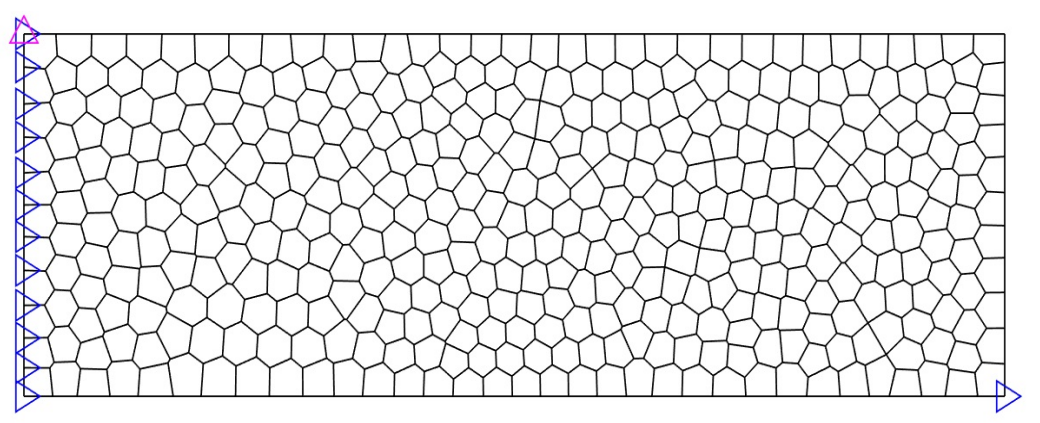

Figura 4.4: Malha gerada com 400 elementos usando o código Polytop [19] 


\section{- Problema de One-node Connections}

Este problema é encontrado, em geral, com elementos triangulares e quadrilaterais onde a conectividade entre elementos ocorre em torno de um nó, conforme ilustrado na Figura 4.5.

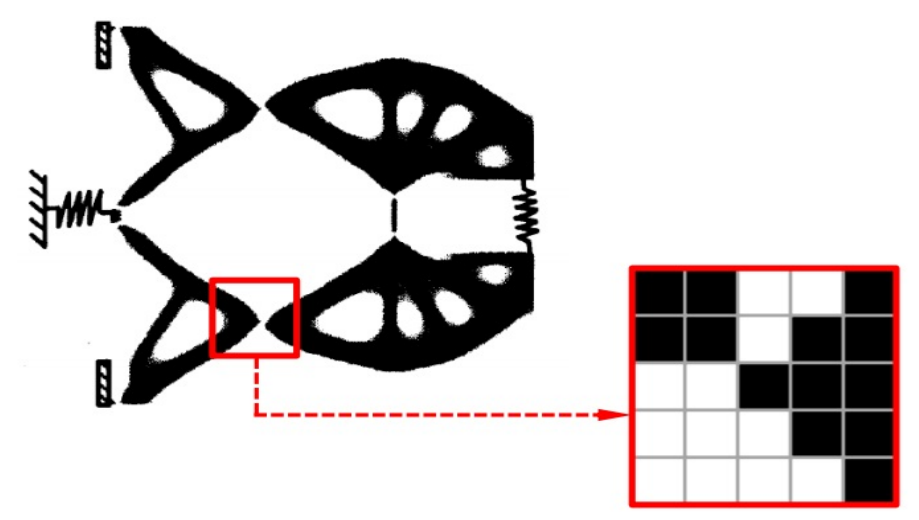

Figura 4.5: Problema One-Node Connection

Utilizando-se elementos poligonais, a malha irá possuir elementos conectados por arestas ao invés de nós, reduzindo as instabilidades matemáticas. Assim, agora será explicado o funcionamento do código e as topologias finais geradas por ele. 


\section{2}

\section{Implementação}

Como mencionado anteriormente, a determinação da derivada topológica é um processo matematicamente complexo, porém, sua aplicação é um procedimento simples e se constitui em uma alternativa viável para a otimização topológica clássica. Voltando-se à equação da derivada topológica dada pela Eq. (3.4.20), pode-se escrever:

$$
\psi(\hat{\boldsymbol{x}})=\frac{3}{2} \boldsymbol{\sigma}(\boldsymbol{u}(\hat{\boldsymbol{x}})): \nabla \boldsymbol{u}^{s}(\hat{\boldsymbol{x}})
$$

Utilizando o método SIMP, as densidades intermediárias encontradas ao se fazer a otimização topológica se tornam $\gamma=1$ ou $\gamma=0$, pois a função ultilizada classicamente neste método é:

$$
f(\gamma)=\gamma^{p}
$$

onde p é o parametro de penalização, que no código PolyTop varia de $p=1,0$ a $p=4,0$, com acréscimos de $p=0,5$, i.e., densidades intermediárias, ao serem elevadas a um exponencial elevado, irão tender a zero caso seus valores sejam pequenos, ou a um, caso seus valores sejam grandes, ou seja, no final a estrutura não apresentará densidades intermediárias indesejáveis. Por fim, a nova função a ser minimizada é:

$$
\psi(\hat{\boldsymbol{x}})=\gamma^{p} \frac{3}{2} \boldsymbol{\sigma}(\boldsymbol{u}(\hat{\boldsymbol{x}})): \nabla \boldsymbol{u}^{s}(\hat{\boldsymbol{x}})
$$

Pode-se perceber que existem apenas dois parâmetros a serem determinados para se calcular o valor da derivada topológica em cada elemento: as deformações $\left(\nabla \boldsymbol{u}^{s}\right)$ e os campos de tensão $(\boldsymbol{\sigma})$. A partir do código PolyTop é necessário criar uma função que irá gerar o vetor de valores da função a ser minimizada. Esta função é apresentada a seguir: 


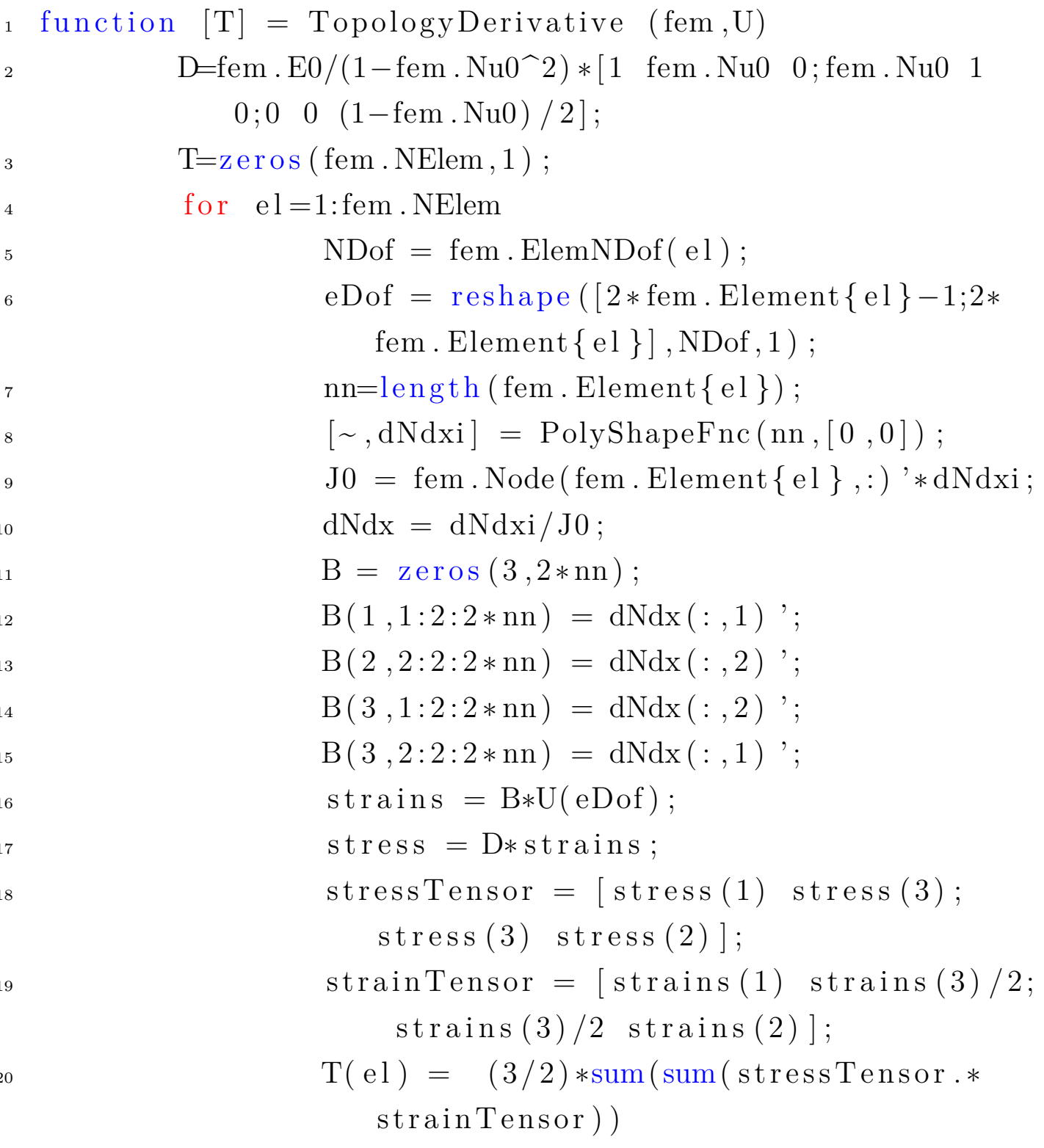

end 
As tensões e deformações nodais podem ser obtidas como:

$$
\begin{gathered}
\sigma=D \epsilon \\
\epsilon=B d .
\end{gathered}
$$

A matriz constitutiva $\boldsymbol{D}$ depende do módulo de Young E e do coeficiente de Poisson $\nu$. A linha 2 do código mostra como é feita esta operação. A expressão da matriz $\boldsymbol{D}$ é:

$$
\boldsymbol{D}=\frac{E}{1-\nu^{2}}\left[\begin{array}{ccc}
1 & \nu & 0 \\
\nu & 1 & 0 \\
0 & 0 & \frac{(1-\nu)}{2}
\end{array}\right]
$$

A matriz "deformação-deslocamento" $(\boldsymbol{B})$ é calculada a partir das funções de forma $(\boldsymbol{N})$, obtida a partir da função PolyShapeFnc (linha 8), a qual recebe o número de nós do elemento e a coordenada do centro do elemento. As funções de forma são dadas por:

$$
N_{i}(\boldsymbol{\xi})=\frac{\alpha_{i}(\boldsymbol{\xi})}{\sum_{j=1}^{n} \alpha_{j}(\boldsymbol{\xi})}
$$

onde i é o número de nós que varia de $1 \leq i \leq n$, e $\alpha_{i}(\xi)$ são os interpoladores de forma, dados por:

$$
\alpha_{i}(\boldsymbol{\xi})=\frac{A\left(p_{i-1}, p_{i}, p_{i+1}\right)}{A\left(p_{i-1}, p_{i}, \boldsymbol{\xi}\right) A\left(p_{i}, p_{i+1}, \boldsymbol{\xi}\right)}
$$

onde $\mathrm{A}(\mathrm{a}, \mathrm{b}, \mathrm{c})$ é a área do triângulo definido pelos vértices $\mathrm{a}$, b e c como ilustrado na Figura 4.6a:

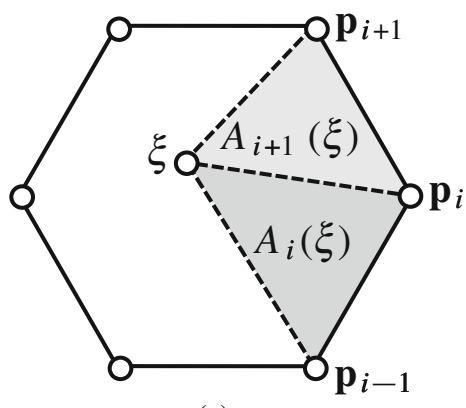

(a)

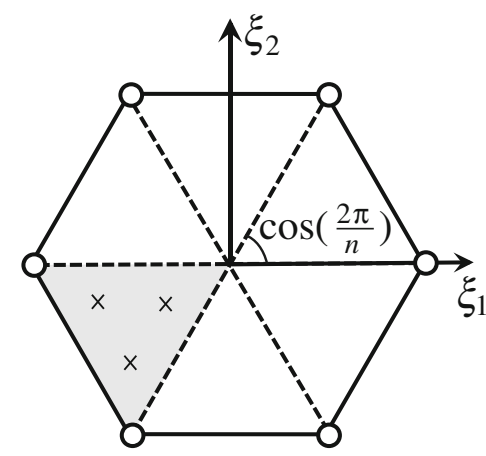

(b)

Figura 4.6: (a) área dos triângulos; (b) sistema de coordenadas. Fonte: [19] 
O desenvolvimento destas equações podem ser vistas em [19]. A partir das funções de forma, calcula-se a Jacobiana (Linha 9), para finalmente chegar-se na matriz de deformação-deslocamento dada por:

$$
\boldsymbol{B}=\left[\begin{array}{ccccccc}
\frac{\partial N_{1}}{\partial \xi_{1}} & 0 & \frac{\partial N_{2}}{\partial \xi_{1}} & 0 & \ldots & \frac{\partial N_{n}}{\partial \xi_{1}} & 0 \\
0 & \frac{\partial N_{1}}{\partial \xi_{2}} & 0 & \frac{\partial N_{2}}{\partial \xi_{2}} & \ldots & 0 & \frac{\partial N_{n}}{\partial \xi_{2}} \\
\frac{\partial N_{1}}{\partial \xi_{2}} & \frac{\partial N_{1}}{\partial \xi_{1}} & \frac{\partial N_{2}}{\partial \xi_{2}} & \frac{\partial N_{2}}{\partial \xi_{1}} & \ldots & \frac{\partial N_{n}}{\partial \xi_{2}} & \frac{\partial N_{n}}{\partial \xi_{1}}
\end{array}\right]
$$

Com todos os termos definidos, calculam-se as tensões e as deformações a partir das Eqs. (4.2.4) e (4.2.5), definidas na forma matricial por:

$$
\begin{gathered}
\boldsymbol{\sigma}=\left[\begin{array}{cc}
\sigma_{x} & \sigma_{x y} \\
\sigma_{x y} & \sigma_{y}
\end{array}\right] \\
\boldsymbol{\epsilon}=\left[\begin{array}{cc}
\epsilon_{x} & \frac{\epsilon_{x y}}{2} \\
\frac{\epsilon_{x y}}{2} & \epsilon_{y}
\end{array}\right] .
\end{gathered}
$$

As tensões e as deformações são representadas pelas variáveis strains e stress nas linhas 18 e 19 .

Lembrando que a derivada topológica é calculada para cada elemento e no centro dos mesmos, podemos relacionar os cálculos apresentados anteriormente da seguinte forma:

Linha(as)

2: Cálculo da matriz de elasticidade

3: Inicialização do vetor da derivada topológica

4-10: Cálculo das funções de forma

11-15: Cálculo da matriz de deformação-deslocamento

16-19: Cálculo das matrizes de tensão e deformação

20-22: Cálculo da derivada topológica

Com o vetor de valores da derivada topológica para cada elemento obtido, basta fazer uma pequena alteração no código original do PolyTop para substituir o cálculo da compliance pelo cálculo da derivada topológica, tal como mostrado no Pseudo-código abaixo: 


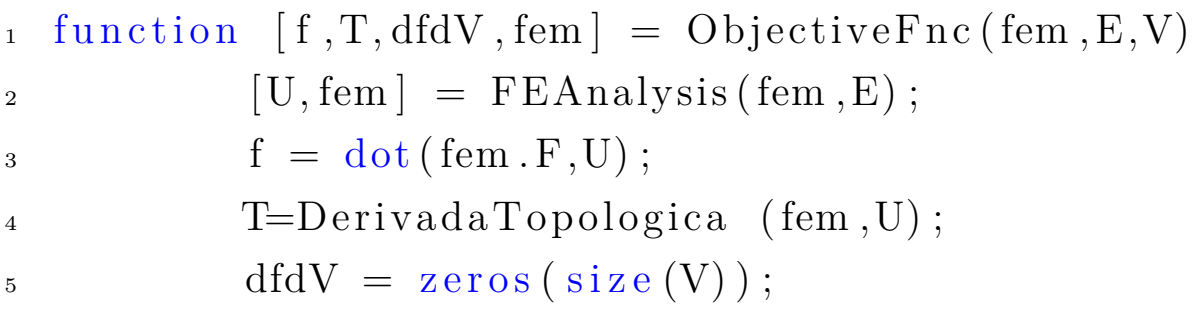

Assim, o processo pode ser interpretado da seguinte forma:

1. Iniciar o array de densidades $\gamma=0,5$ como chute inicial para o problema minimização e inicializar o fator de penalidade $p=1$.

2. Calcular os valores de tensão e deformação.

3. Utilizar o OC como método para determinar a nova distribuição de material assim como [19].

4. Repetir os itens 2 e 3 até que o novo valor da energia interna seja igual ou menor a um erro de 1e-4, ou que o número de iterações chegue a 150.

5. Após a primeira convergência, alterar o valor de penalidade $p$ para $p=p+0,5$ e usar o array de $\gamma$ obtido no processo de penalidade anterior como chute inicial.

6. Repetir os processos de 2-5 até que o valor de penalidade chegue a $p=4$. 
5

\section{Resultados}

\section{1}

Metodologia

Para a obtenção de resultados serão trabalhados os seguintes casos:

- Messerschmitt-Bölkow-Blohm Domain (MBB Domain)

- Hook Domain

- Serpentine Domain

Todos os domínios serão discretizados com um total de 8000 elementos, o volume final será de $40 \%$ do volume inicial e o raio de busca $\mathrm{M}=0,2$. Desta maneira teremos um domínio simples (MBB) e dois domínios complexos (Hook e serpentine), e com estes resultados pode-se comparar suas topologias finais com algumas de diversos autores. 


\section{2}

\section{MBB Domain}

Para o primeiro problema, considera-se uma viga bi-apoiada onde seu comprimento possui valor $L=4$, altura $h=1$ e uma força concentrada de $F=2$ em seu centro, ou seja, F se encontra em $L=2$ e $h=1$. Para as propriedades do material, considera-se o Módulo de Young $E=1$ e o coeficiente de poisson $\nu=\frac{1}{3}$, e, devido as simetrias, considera-se apenas metade da estrutura. Desta forma a otimização topológica, utilizando o método da derivada topológica, chega ao resultado da Figura 5.1.

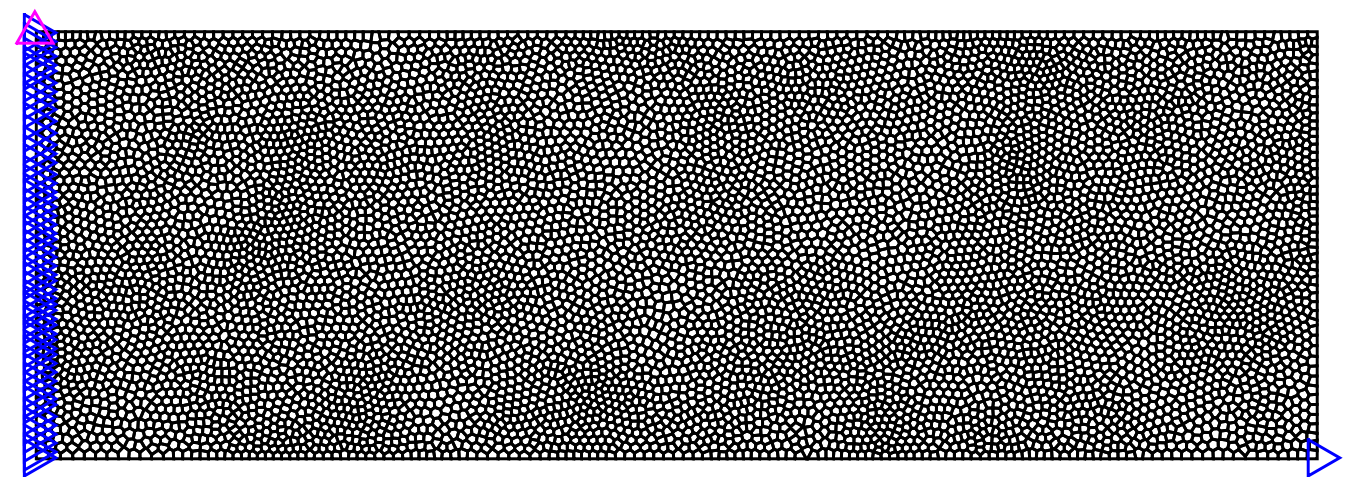

(a)

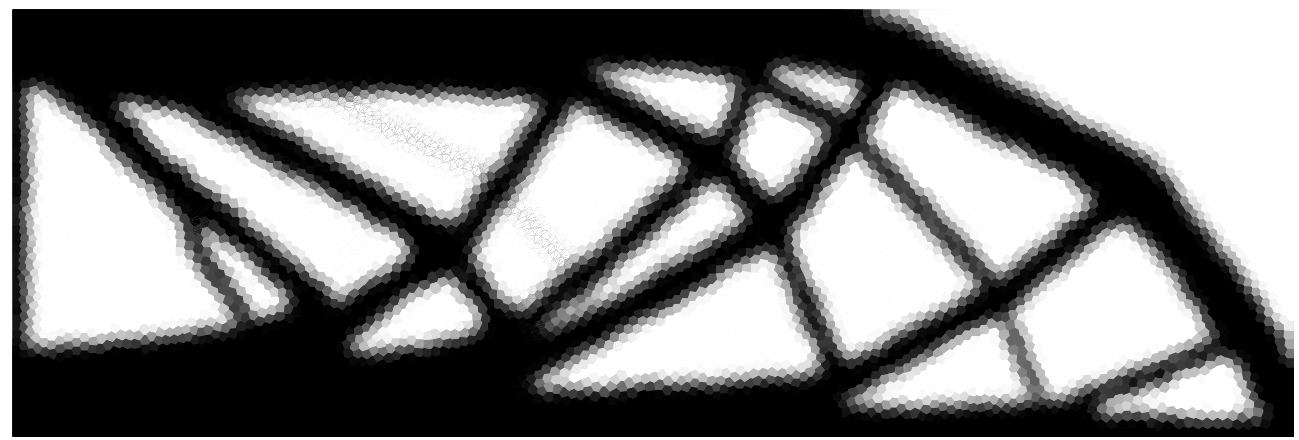

(b)

Figura 5.1: (a) Malha utilizada para a realização da otimização topológica; (b) Topologia final obtida após $p=4$.

Na Figura 5.1a, os triângulos azuis representam pontos em que a estrutura possui um engaste, enquanto que os triângulos rosas reprensentam pontos em que há uma força atuante. Pela Figura 5.1b, pode-se observar uma topologia final semelhante a de vários autores $[4,13,14]$. 


\section{3}

\section{Hook Domain}

Uma vantagem dos elementos poligonais é que estes permitem uma maior flexibilidade na discretização de domínio complexos. O domínio hook, muito usado em guindastes para mover cargas elevadas, pode ser otimizado pelo método da derivada topológica, chegando em uma melhor distribuição de material. Considerando este domínio onde, o raio de onde se encontra o carregamento possui valor $R=0,80$, o raio da região em que se encontra engastada possui valor $r=0,50$, um carregamento de $\bar{F}=15$ e as mesmas propriedades do material do exemplo anterior. Utilizando novamente o método da derivada topológica, encontramos o resultado da Figura 5.2.

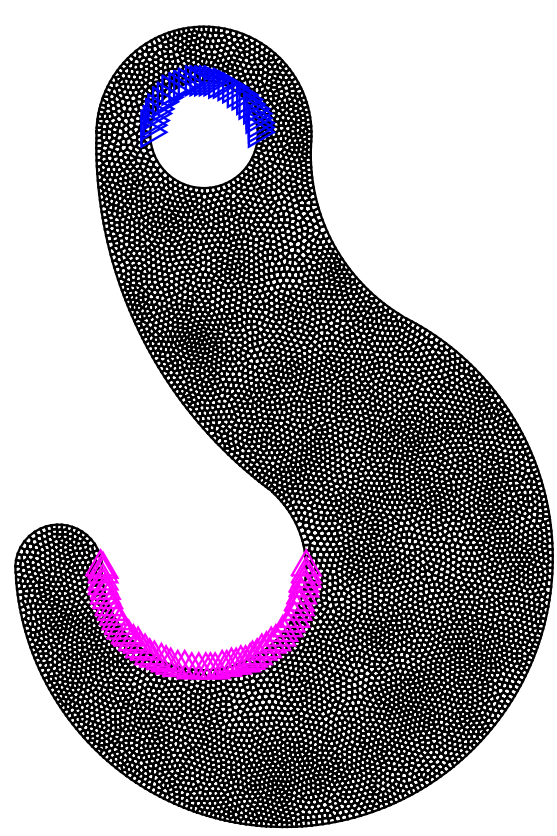

(a)

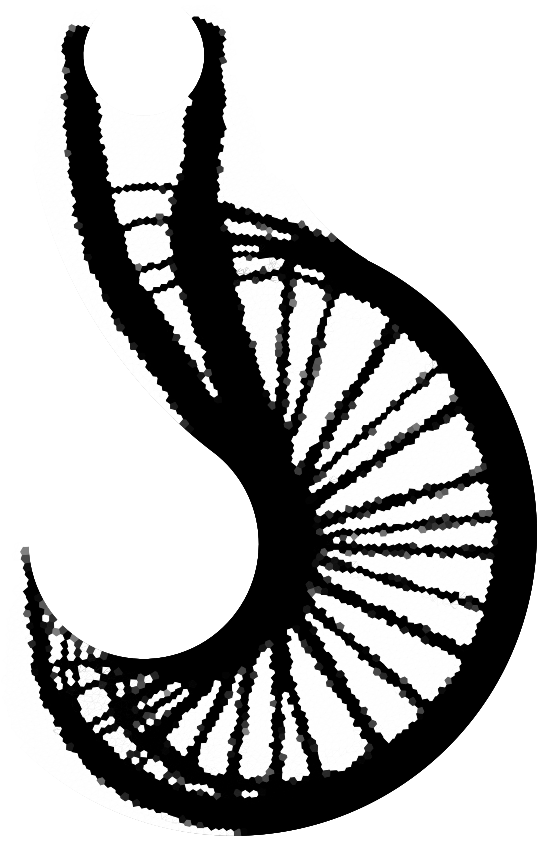

(b)

Figura 5.2: (a) Malha utilizada para a realização da otimização topológica; (b) Topologia final obtida após $p=4$.

A Figura 5.2a ilustra esta malha mais complexa, enquanto que a Figura $5.2 \mathrm{~b}$ ilustra a topologia final obtida após o processo iterativo. 


\section{4}

\section{Serpentine Domain}

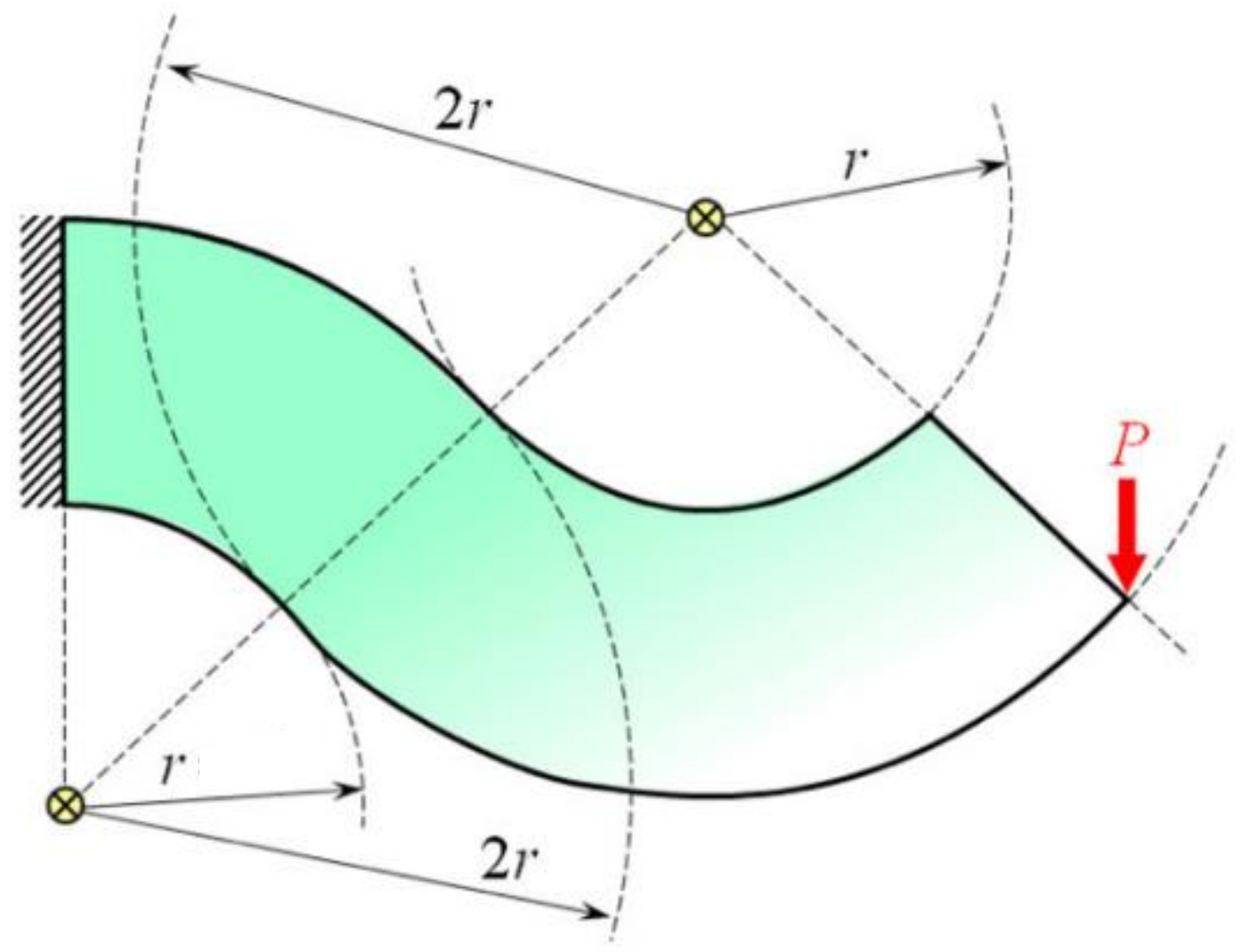

Figura 5.3: Domínio serpentine [31]

Para o terceiro caso, considera-se o domínio serpentine ilustrado na Figura 5.3 acima. Considerando um raio de curvatura igual a $r=0,25$ e uma força $P=5$ concentrada em sua ponta, a otimização topológica conduz ao resultado da Figura 5.4. 

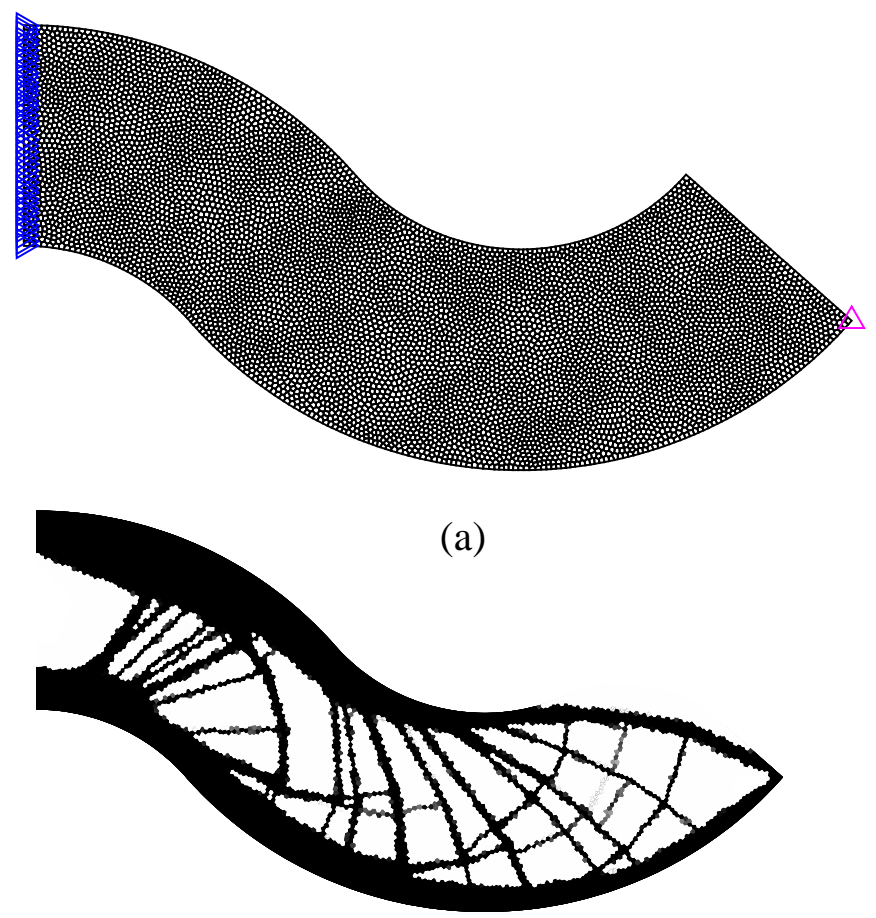

(b)

Figura 5.4: (a) Malha utilizada para a realização da otimização topológica; (b) Topologia final obtida após $p=4$.

Comparando a topologia final obtida com [31], pode-se observar resultados bastante parecidos, indicando que o método de aproximação via derivada topológica possui topologias condizentes com os obtidos por outros autores. 


\section{6 \\ Conclusões}

A derivada topológica é um tópico de pesquisa relativamente novo, mas simples de ser aplicada em problemas de otimização topológica. Foi possível observar que a dedução para se obter a expansão assintótica topológica é matematicamente complexa, mas, por outro lado, com a ajuda de vários trabalhos já existentes, o processo de se encontrar a forma da expansão assintótica topológica se torna mais simples.

Aplicando este conceito a malhas com mais de 8000 elementos, o resultado se mostrou bastante satisfatório, principalmente com elementos de ordens superiores. Este conceito, embora tenha tido foco neste trabalho na otimização topológica, pode ser aplicado em diferentes áreas de conhecimento, porém, é interessante ressaltar que, independentemente da aplicação, a ideia é sempre obter a forma da expansão assintótica topológica da Eq. (2.1.1) que levará à derivada topológica.

Espera-se que trabalhos futuros na área de otimização topológica possam ser realizados usando a derivada topológica, não apenas para estruturas 2D, mas também 3D, visto que a derivação matemática para se obter a forma da derivada topológica para o caso 3D é similar. Algumas possibilidades de trabalhos futuros podem ser: implementar no código MATLAB, desenvolvido neste trabalho, o efeito da temperatura na estrutura e incluir restrições locais de tensão no problema de otimização topológica. 


\section{Referências bibliográficas}

[1] D. E. GOLDBERG; M. P. SAMTANI. Engineering optimization via genetic algorithm. 9th Conf. Electronic Computation, New York:471-482, 1991.

[2] A. C. C. LEMONGE. Aplicação de algoritmos genéticos em otimização estrutural. tese (doutorado) - coppe, programa de engenharia civl. universidade federal do rio de janeiro. 1999.

[3] O. SIGMUND; M. P. BENDSOE. Topology Optimization - Theory, Methods and Applications. Springer, Berlin, 2004.

[4] O. SIGMUND. A 99 line topology optimization code written in matlab. Structural and Multidisciplinary Optimization, 21:120-127, 2001.

[5] K. SVANBERG; M. WERME. Topology optimization by sequential integer linear programming. Solid Mechanics and Its Applications, 137:425-436, 2006.

[6] J. A. SETHIAN AND P. SMEREKA. Level set methods for fluids interfaces. Annual Review of Fluid Mechanics, 35:341-372, 2003.

[7] A. SHARMA. Level set method for computational multi-fluid dynamics: A review on developments, applications and analysis. Sãdhanã, 40:627-652, 2015.

[8] G. ALLAIRE; F. JOUVE; A. TOADER. Structural optimization using sensitivity analysis and a level-set method. Journal of Computational Physics, 194:363-393, 2004.

[9] A. SHARMA. A topology optimization method based on the level set method incorporating a fictitious interface energy. Computer Methods in Applied Mechanics and Engineering, 199:2876-2891, 2010.

[10] J. J. KINGMAN; K. D. TSAVDARIDIS; V. V. TOROPOV. Applications of topology optimization in structural engineering. University of Leeds, 42:665-679, 2014. 
[11] A. ZOCHOWSKI; J. SOKOLOWSKI. On the topological derivative in shape optimization. SIAM Journal on control and Optimization, 37:1251-1272, 1999.

[12] A. A. NOVOTNY. Análise de sensibilidade topológica - teoria e aplicações. Notas em matemática aplicada, 73, 2014.

[13] K. SURESH. A 199-line matlab code for pareto-optimal tracing in topology optimization. Structural and Multidisciplinary Optimization, 42:665-679, 2010.

[14] M. OTOMORI; T. YAMADA; K. IZUI; S. NISHIWAKI. Matlab code for a level set-based topology optimization method using a reaction diffusion equation. Structural and Multidisciplinary Optimization, 51:1159-1172, 2015.

[15] M. Y. WANG. Topology Optimization with Level Set Method Incorporating Topological Derivative. 6th World Congresses of Structural and Multidisciplinary Optimization

[16] D. E. CAMPEAO; S. M. GIUSTI; A. A. NOVOTNY. Topology Design of Plates Considering Different Volume Control Methods. Engineering Computations, 2013.

[17] S. AMSTUTZ; A. A. NOVOTNY; N. V. GOETHEM. Topological sensitivity analysis for elliptic differential operators of order $2 \mathbf{m}$. Journal of Differential Equations, 256:1735-1770 2014.

[18] A. J. TORII; A. A. NOVOTNY; R. B. SANTOS. Robust compliance topology optimization based on the topological derivative concept. International Journal for Numerical Methods in Engineering; 114:660-674, 2018.

[19] C. TALISCHI AND G. H. PAULINO AND A. PEREIRA AND IVAN F. M. MENEZES. Polytop: a matlab implementation of a general topology optimization framework using unstructured polygonal finite element meshes. Structural and Multidisciplinary Optimization, 45:329-357, 2012.

[20] V. A. KOZLOV; V. G. MAZ'YA; A. B. MOVCHAN . Asymptotic analysis of fields in multi-structures. Oxford: Clarendon Press, 1999.

[21] T. MURA. Micromechanics of defects in solids. Dordrecht: Kluwer academic publishers, 1987. 
[22] M. ITSKOV. On the theory of fourth-order tensors and their applications in computational mechanics. Comput. Methods Appl. Mech. Engrg., 189:419-438, 2000.

[23] V. SHOBEIRI. Structural Topology Optimization Based on the Smoothed Finite Element Method. Latin American Journal of Solids and Structures, 13:378--390, 2016.

[24] M. REZAIEE-PAJAND; M. YAGHOOBI. A robust triangular membrane element. Latin American Journal of Solids and Structures, 11:26482671, 2014.

[25] A. L. GAIN; G. H. PAULINO. Phase-field based topology optimization with polygonal elements: a finite volume approach for the evolution equation. Structural and Multidisciplinary Optimization, 46:327-342, 2012.

[26] C. TALISCHI; G. H. PAULINO; A. PEREIRA; I. F. M. MENEZES. Polygonal finite elements for topology optimization: A unifying paradigm. International Journal for Numerical Methods in Engineering, 82:671-698, 2010.

[27] C. TALISCHI; G. H. PAULINO; A. PEREIRA; I. F. M. MENEZES. Polygonal finite elements for topology optimization: A unifying paradigm. International Journal for Numerical Methods in Engineering, 82:671-698, 2010.

[28] P. A. BROWNE. Topology Optimization of Linear Elastic Structures. 2013.

[29] D. BOFFI; F. BREZZI; M. FORTIN. Mixed Finite Element Methods and Applications. 2013.

[30] A. SHUKLA; A. MISRA; S. KUMAR. Checkerboard problem in finite element based topology optimization. International Journal of Advances in Engineering \& Technology, 6(4):1769-1774, 2013.

[31] H. NGUYEN-XUAN. A polytree-based adaptive polygonal finite element method for topology optimization. International Journal for Numerical Methods in Engineering, 110:972-1000, 2017. 